\title{
Regulation of class Ila HDAC activities: it is not only matter of subcellular localization
}

In response to environmental cues, enzymes that influence the functions of proteins, through reversible post-translational modifications supervise the coordination of cell behavior like orchestral conductors. Class Ila histone deacetylases (HDACs) belong to this category. Even though in vertebrates these deacetylases have discarded the core enzymatic activity, class Ila HDACs can assemble into multiprotein complexes devoted to transcriptional reprogramming, including but not limited to epigenetic changes. Class Ila HDACs are subjected to variegated and interconnected layers of regulation, which reflect the wide range of biological responses under the scrutiny of this gene family. Here, we discuss about the key mechanisms that fine tune class Ila HDACs activities.

First draft submitted: 8 September 2015; Accepted for publication: 29 October 2015; Published online: 21 January 2016

Keywords: AMPK • FOXO • HDAC4 • HDAC5 • HDAC7 • HDAC9 • LKB • MEF2 • PKD - SIK

\section{The class Ila HDACs}

Lysine acetylation is a post-translational modification (PTM) that, when exercised on histones, plays a key role in marking transcriptionally active genomic regions. Similarly to other PTMs, acetylation is not restricted to chromatin remodeling but it can modulate different molecular machineries, which control cell cycle progression, actin nucleation, splicing and nuclear transport [1].

Lysine-acetylation homeostasis is under the supervision of two families of enzymes, with antagonistic activities: the histone acetyl transferases and the histone deacetylases (HDACs). HDACs repertoire in mammals comprises 18 genes that can be grouped into five subfamilies on the basis of their sequence homology and phylogenetic criteria [2]. To the subclass IIa belong HDAC4, HDAC5, $H D A C 7$ and $H D A C 9$ (for an excellent review on HDACs see [2]). These proteins share specific and characteristic features, which are highlighted in Box 1.
Class IIa HDACs as part of multiprotein complexes are involved in the regulation of assorted cellular responses. They generally act at the apex of specific genetic programs, by influencing the landscape of gene expressed in a specific context. Although principally investigated as regulators of transcription and in particular of myocyte enhancer factors (MEF2) transcription factors, alternative partners and functions, in particular when localized in the cytoplasm, cannot be excluded (for a discussion on some class IIa HDACs partners see [3]).

Similarly to other epigenetic modifiers, class IIa HDACs do not recognize the DNA, instead by interacting with a selected number of transcription factors, they are recruited on specific genomic regions in a sequencedependent manner [2]. During embryonic development, class IIa HDACs are actively involved in controlling specific differentiation pathways and tissue morphogenesis. In adult tissue they are part of several adaptive responses (for recent reviews see $[4,5]$ ).
Epigenomics

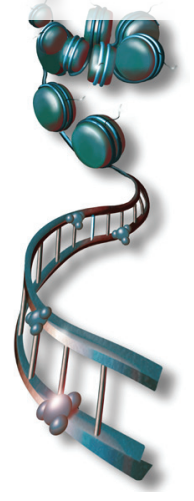

Eros Di Giorgio' \& Claudio Brancolini ${ }^{*, 1}$

'Department of Medical \& Biological Sciences, Università degli Studi di Udine, P.le Kolbe 4 - 33100 Udine, Italy *Author for correspondence: Tel.: +390432494382

claudio.brancolini@uniud.it
Future
Medicine
part of 


\section{Box 1. Class Ila histone deacetylases hallmarks.}

- An extended amino-terminal region involved in the interaction with several partners and different transcription factors, which recruits these repressors on specific genomic regions

- A carboxy-terminal deacetylase domain that, in vertebrates, is enzymatically 'inactive' (or at least inactive vs acetyl-lysine)

- The co-presence of nuclear localization sequences and of nuclear export sequences, which confers environmental signaling regulated nuclear/cytoplasmic shuttling

- Interactions with several partners, comprising multiprotein complexes recruiting class I histone deacetylases, which confer the deacetylase activity

Not surprisingly, these arenas of copious interventions find correspondence in the multiple options available to oversee class IIa HDACs activities. In this review, we will discuss about these different options and how cells and the microenvironment govern class IIa HDACs activities.

\section{Transcription is the first decision: when \& where}

Paradoxically, although the tissue-specific expression of class IIa HDACs was immediately considered a distinctive trait of this subfamily [4], a limited number of studies have addressed the control operated at the level of transcription on these genes. Apparently, class IIa HDACs transcription is under the control of many signaling pathways.

\section{Genomic organization}

The complexity of such regulation is mirrored by the existence of several transcriptional variants. A summary of the genomic organization of the different class IIa HDACs is shown in Table 1. Concerning $H D A C 4$ only a single isoform has been described up to now, composed by 26 coding exons and 1 noncoding exon (mRNA $8980 \mathrm{bp}$ and ORF/open reading frame, 3252 bp-long) [6]. The reference sequence of the longer RNA encoded by HDAC5 locus is 5324 bp and produces a 3369 bp ORF that comprises all coding exons of its paralog $H D A C 4$ [6]. A second described transcription variant of $H D A C 5$ lacks exons 14 and 15 and produces a protein of 75 amino acids (aa) shorter, characterized by a deficient catalytic domain [6]. Two different splicing isoforms of $H D A C 7$ have been characterized [6,7]: the most common spliced isoform is made up of 25 exons and an unspliced isoform that retains the first intron after the first ATG [7]. This unspliced mRNA could in principle produce two products. A small peptide of 7 aa and a protein of 22 aa shorter compared with the common form [7]. In this case a second ATG proficient in translation is used [7]. The unspliced and spliced isoforms differentially influence cell proliferation and their generation is regulated during vascular smooth muscle cells (VSMCs) differentiation [7,8]. Curiously, the last 612 bp of HDAC7 are antisense respect to the
SLC48A1 gene, raising the possibility of an involvement in controlling the translation of this gene [6].

The canonical isoform of HDAC9 is made up of 23 coding exons, which are translated into a 1011 aa polypeptide [6,9]. A well-known variant of $H D A C 9$ is called MITR or HDAC9DCD (HDRP/MITR) [10]. It generates a shorter protein $(593 \mathrm{aa})$ lacking the deacetylase domain. This variant is not a simple truncation of full-length $H D A C 9$, because it retains 16 unique residues codified by intron 12 which are $3^{\prime}$ to the canonical splice donor site [9]. Despite the truncation, MITR represses MEF2-dependent transcription [10].

Removal of exon 7 during splicing generates a protein (HDAC9Dexon7) which is 37 aa shorter and that lacks two serines (Ser223 and Ser253) involved in the nuclear export and a portion of the nuclear localization signal. Another exon that may be removed is the twelfth. The corresponding protein is 981 aa long and, since the exon 12 contains the SUMOylation site, this splice variant cannot be SUMOylated [9,11]. Additional HDAC9 splicing variants include an isoform lacking both the seventh and the twelfth exons [9] and a deletion of a portion of the 15th exon, which generates similarly to $M I T R$, a protein deprived of the deacetylase domain [9].

\section{Transcriptional control}

Experimentally, the expression of HDAC4 can be repressed by mithramycin [12], which after binding to GC-rich sequences displaces the $\mathrm{Sp}$ transcription factors [13]. Binding of Sp1 and Sp3 to the HDAC4 promoter was confirmed by EMSA and ChIP experiments and the manipulation of Sp1 and Sp3 expression was coupled to a parallel variation of HDAC4 expression [12]. Furthermore, the antineoplastic properties of the turmeric root derivative curcumin seem to partially depend on the inhibition of the Sp1 action on HDAC4 promoter [14].

Also HDAC7 transcription seems to be under the control of Sp1. In particular, it was reported that Sp1 stimulates the transcription of HDAC7 mRNAs during the PDGF-BB-induced differentiation of murine embryonic stem cells into smooth-muscle cells (SMCs) [15]. HDAC7 expression is similarly induced by PDGF-BB also in VSMCs [16]. 
In addition to Sps, class IIa HDACs transcription must depend on additional circuits, operating in a tissue-specific manner. For example, HDAC4 expression is induced after denervation in atrophic muscles, where it influences a metabolic shift [17]. A feed-forward mechanism is involved in such regulation. The initial nuclear relocalization of HDAC4 in atrophic muscles causes the activation of myogenin, which in turn induces HDAC4 transcription, thus alimenting the expression of the deacetylase [17].

In bones, HDAC4 is the highest expressed class IIa HDACs and it plays an irreplaceable role in the process of chondrocyte hypertrophy [18]. During skeletogenesis, the levels of HDAC4 increase in murine prehypertrophic chondrocytes in the growth plate at E18.5, while HDAC4 is not expressed at detectable levels in proliferating chondrocytes, in bones and osteoblasts [18]. The contribution of the transcriptional machinery in this switch is unknown.

Similarly, $H D A C 7$ is highly expressed in pre-B cells but dramatically downregulated, both at RNA and protein levels, during lineage conversion to macrophages [19]. Although HDAC7 repression is necessary for the transcription of MEF2-target genes, which are important for macrophage functions, the mechanisms that control HDAC7 levels still need to be investigated [19].

In embryonic stem cells Oct3/4 prevent HDAC4mediated repression of stemness, by binding the first intron of the deacetylase and thus interfering with splicing maturation [20]. This regulation was hypothesized to limit the negative influence of class IIa HDACs on stemness, possibly through the repression of Oct3/4 and Klf4 genes.

Upregulation of class IIa HDACs transcription is observed during muscle differentiation, as part of a negative-feedback loop, to fine tuning the rate of differentiation. In this case MEF2A, MEF2C and MEF2D are able to bind the promoter of Hdac9 and to induce its expression [21]. It is not clear at the moment whether MEF2s regulate directly also the transcription of other class IIa HDACs (see below).

The control operated by MEF2s on class IIa transcription could also be involved in the compensatory responses observed in different experimental settings. In certain cell lineages when a class IIa HDAC member is depleted another member is upregulated [22-24]. Once MEF2 get rid of a class IIa HDACs member, they might transcribe other members of the family, by binding to the proximal promoter (in case of $H D A C 5$ and $H D A C 9$ ) or to the enhancer (for HDAC4 and HDAC7; see below).

Although few information are available on the transcriptional control operated on class IIa HDACs, the ENCODE project disposes of the ChIP-Seq data for several transcription factors (TFs) and other elements controlling transcription [25]. Numerous elements including TFs, epigenetic modifiers and architectural proteins bind the proximal promoter of $H D A C 4$ and $H D A C 7$, thus suggesting that transcription of these two deacetylases is under intense supervision (Figure 1). Less covered are the proximal promoters of HDAC5 and of HDAC9. Binding of MEF2 TFs was confirmed in the proximal promoter of $H D A C 9$ (Figure 2). MEF2s bind also the HDAC5 proximal promoter, hence also this deacetylase can be under control of MEF2s as part of a negative feedback loop [24]. In general, the complexity of TFs and epigenetic regulators assembling onto the promoters of class IIa HDACs testifies the importance of the transcriptional control in the homeostasis of this gene family.

Different proto-oncogenes and cell cycle supervisors bind the promoters of class IIa HDACs (Figures $1 \& 2$ ). Interestingly, in support of an involvement of $H D A C 4$ and HDAC7 in the processes of oncogenic transformation [26], some proto-oncogenes such as JUN, FOS, MYC, which control the G0/G1 transition, bind the proximal promoters of these deacetylases [25]. These

Table 1. Class Ila histone deacetylases genomic organization.

\begin{tabular}{|c|c|c|c|c|c|c|c|}
\hline $\begin{array}{l}\text { Histone } \\
\text { deacetylases }\end{array}$ & Localization & Extension & TSSs & SV & mRNA & Exons & Longer ORF \\
\hline HDAC4 & $\begin{array}{l}\text { Chr 2q37.3 minus } \\
\text { strand }\end{array}$ & 353479 & 7 & 16 s 6 uns & 1 & $\begin{array}{l}27 \\
(26 c / 1 n c)\end{array}$ & 3256 \\
\hline HDAC5 & $\begin{array}{l}\text { Chr 17q21 minus } \\
\text { strand }\end{array}$ & 46893 & 8 & 17 s 6 uns & 2 & $\begin{array}{l}27 \\
(26 c / 1 n c)\end{array}$ & 3369 \\
\hline HDAC7 & $\begin{array}{l}\text { Chr } 12 q 13.1 \\
\text { minus strand }\end{array}$ & 37890 & 17 & 31 s 5 uns & 2 & 25 & 2856 \\
\hline HDAC9 & $\begin{array}{l}\text { Chr 7p21.1 plus } \\
\text { strand }\end{array}$ & 912579 & 9 & 17 s 6 uns & 6 & 23 & 3033 \\
\hline
\end{tabular}




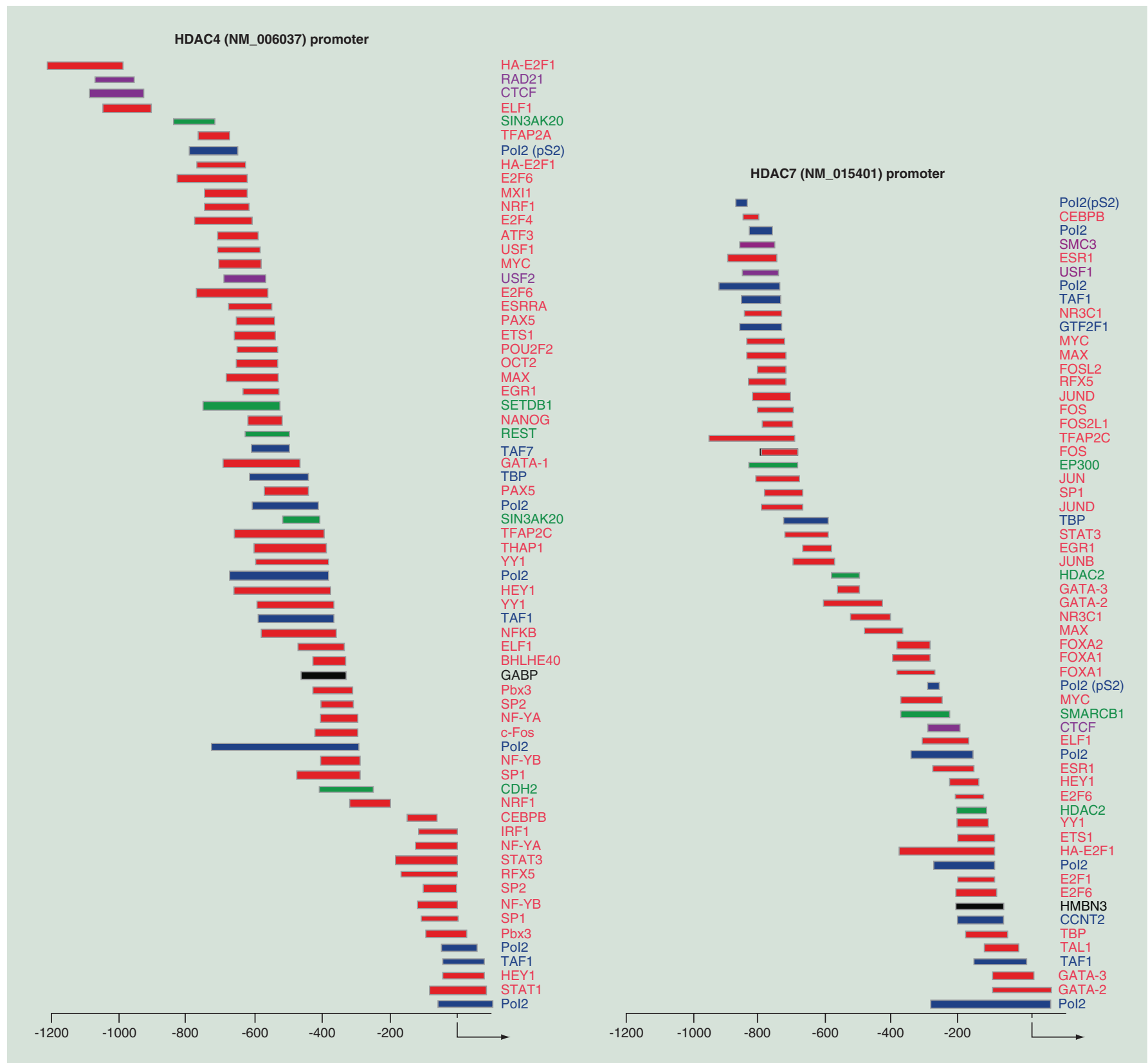

Figure 1. Available ChIP-Seq data from eight different cells lines were analyzed for the binding to the proximal promoters (-1/-1000) of HDAC4 and HDAC7. Transcription factors (red), epigenetic modifiers (green), basic transcriptional machinery (blue), architectural proteins (violet) and their relative region of binding are indicated.

proto-oncogenes could be responsible for the upregulation of $H D A C 7$ mRNA observed in response to PDGF or serum stimulation [15] and of HDAC4 during differentiation of osteoblasts exposed to EGF-like ligands [27]. The ENCODE project finds at least six regulators, which bind the immediate early promoter of all the four class IIa HDACs: Pol2, TAF1, TBP, SP1, YY1, MAX. Not surprisingly, the first three belong to the basal transcription machinery. The presence in this list of $\mathrm{Sp} 1$ confirms the early studies above described. Regarding YY1, it usually binds the chromatin in close proximity of the transcription start site of highly expressed genes [28]. The contemporary presence of MAX and YY1 on the promoters of these deacetylases suggests that class IIa HDACs could be part of the Myc transcription network.

\section{Control of the mRNA stability: miRNAs targeting class Ila HDACs in physiological} contexts

The regulation of the mRNA stability offers another strategy to impact on class IIa HDACs. microRNAs 
targeting these enzymes were initially discovered by studying muscular and chondrocyte differentiation. During myogenesis MEF2, in addition to the negative feedback loop involving $H D A C$, controls also the expression of miR-1, which targets HDAC4 mRNA, thus fueling a positive feedback loop [29,30]. However, this model is complicated by the fact that miR- 1 can also target the 3'UTR of MEF2A [31]. A similar positive feedback loop is operative during neurogenesis. In this case MEF2C promotes the transcription of miR-9, which targets the 3'UTR of HDAC4 [32].

In myoblasts the relative abundance of miR-1 and miR-133 contributes to the equilibrium between proliferation and terminal differentiation [29]. In particular, miR-1 targets the 3'UTR of HDAC4 and promotes myogenesis, while miR-133 destabilizes serum response factor and sustains proliferation. Curiously, the two miRNAs derive from the same polycistronic pre-miRNA and are transcribed together [29]. Importantly, in embryonic hearth and in the somite, MEF2 together with $\mathrm{MyoD}$ positively regulate the transcription of both these miRNAs, by binding to an miR-1/-133 intragenic enhancer [30].

miR-1 is required for the correct fusion of myoblasts but it is also induced by the mTOR pathway and has prohypertrophic properties [33]. miR-1 stimulates also chondrocyte hypertrophy again through HDAC4 inhibition [34]. Another miRNA upregulated during chondrogenesis is miR-365. In particular, miR-365 is mechanosensitive and determines the induction of $\mathrm{Hh}$ and collagen X expression, the latter through the direct targeting of HDAC4 [35]. During osteoblast differentiation, HDAC4 is also regulated by miR-29b, which promotes osteogenesis [36].

TGF-beta represses myogenesis and muscle differentiation. In $\mathrm{C} 2 \mathrm{C} 12$ cells, TGF-beta inhibits muscle differentiation, through the repression of miR-206 and miR-29 and thereby by augmenting HDAC4 expression [37]. Importantly, miR-206 represses hypertrophy of myocytes in vitro but has no effects in the regulation of muscle hypertrophy in vivo, although in both cases it causes a massive drop in HDAC4 levels [37]. Among the plethora of responses regulated by miR-206, it was described that the repression of HDAC4 delays amyotrophic lateral sclerosis progression and promotes regeneration of neuromuscular synapses [38,39]. Finally, miR-206 displays strong tumor-suppressive properties in gastric cancers, again partially due to the repression of HDAC4 [40]. Recently, a cross-talk between the NRF2, HDAC4 and miR-1/miR-206 was described in cancer cells and in vivo tumor models [41]. In KEAP-1-deficient lung cancer cells NRF2 is hyperactive and promotes the reduction of cysteines 667 and 669 in HDAC4 (see below). As a conse- quence, HDAC 4 accumulates in the nucleus $[41,42]$ and represses the transcription of miR-1 and miR-206 [41]. Among miR-1 and miR-206 targets there are key-genes involved in the control of pentose phosphate pathway and tricarboxylic acid cycle [41]. Therefore the activation of NRF2 in cancer cells, at least in part through the HDAC4-mediated repression of miR-1/miR-206, reprograms glucose metabolism toward the pentose phosphate pathway thus providing the substrates needed to support cell growth [41].

Another pathway controlling HDAC4 mRNA stability, via the same miRNAs (MEF2/miR-1/miR-206/ $\mathrm{NOTCH} 3$ ) is active in myoblasts, where it ensures that differentiation takes place with the right timing $[43,44]$. $\mathrm{NOTCH} 3$ delays muscle differentiation at least in part by promoting the dephosphorylation of MEF2. An increase in MEF2 levels is sufficient to overcome this inhibition and to directly transcribe miR-1 and miR-206. In turn, these miRNAs have a double prodifferentiative effect since they target both HDAC4 [29] and $\mathrm{NOTCH} 3$ [43].

Certain studies have proposed a role of HDAC4 in the pathogenesis of Huntington's disease [45,46]. In agreement, targeting of HDAC4 by miR-22 shows neuroprotective effects [47]. miR-22 is upregulated also during myocyte differentiation and cardiomyocyte hypertrophy [47]. Its overexpression is sufficient to induce cardiomyocyte hypertrophy in vivo, while miR-22-null hearts are resistant to stress-induced cardiac hypertrophy [48]. These phenotypes at least in part might be explained by the suppression of HDAC4 [48].

miR-22-mediated silencing of HDAC4 is also involved in the pathogenesis of emphysema [49]. In particular, high levels of miR-22 are present in antigen presenting cells, derived from a murine model of lung emphysema. This upregulation causes a strong decrease in HDAC4 levels and the subsequent increase in the release of IL-6. IL-6 stimulates the activation of the TH17 subset of helper T cells that are responsible for the establishment of a state of chronic inflammation [49].

Finally, some miRNAs regulated during embryogenesis recognize $H D A C 4 \mathrm{mRNA}$, thus abolishing its repressive activities on certain loci. For example in the fetal brain, levels of the epigenetic regulators methyl CpG-binding protein 2 and HDAC4 are kept low by presence of miR-483-5p [50].

While in literature the stability of the $3^{\prime} \mathrm{UTR}$ of $H D A C 4$ has been studied in details, little information are currently available on the regulation of the other class IIa HDACs by miRNAs. Few miRNAs target the 3'UTR of HDAC5 in an exclusive manner. One of them is miR-2861 [51,52]. Similarly and redundantly to miR-1, miR-2861 is a positive regulator of chondrocyte hypertrophy and osteoblast differentiation, through 


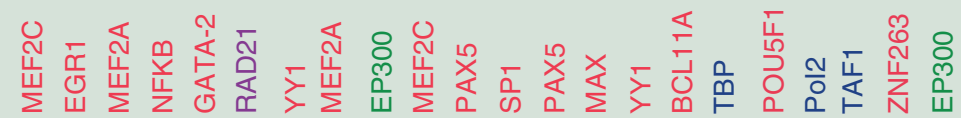

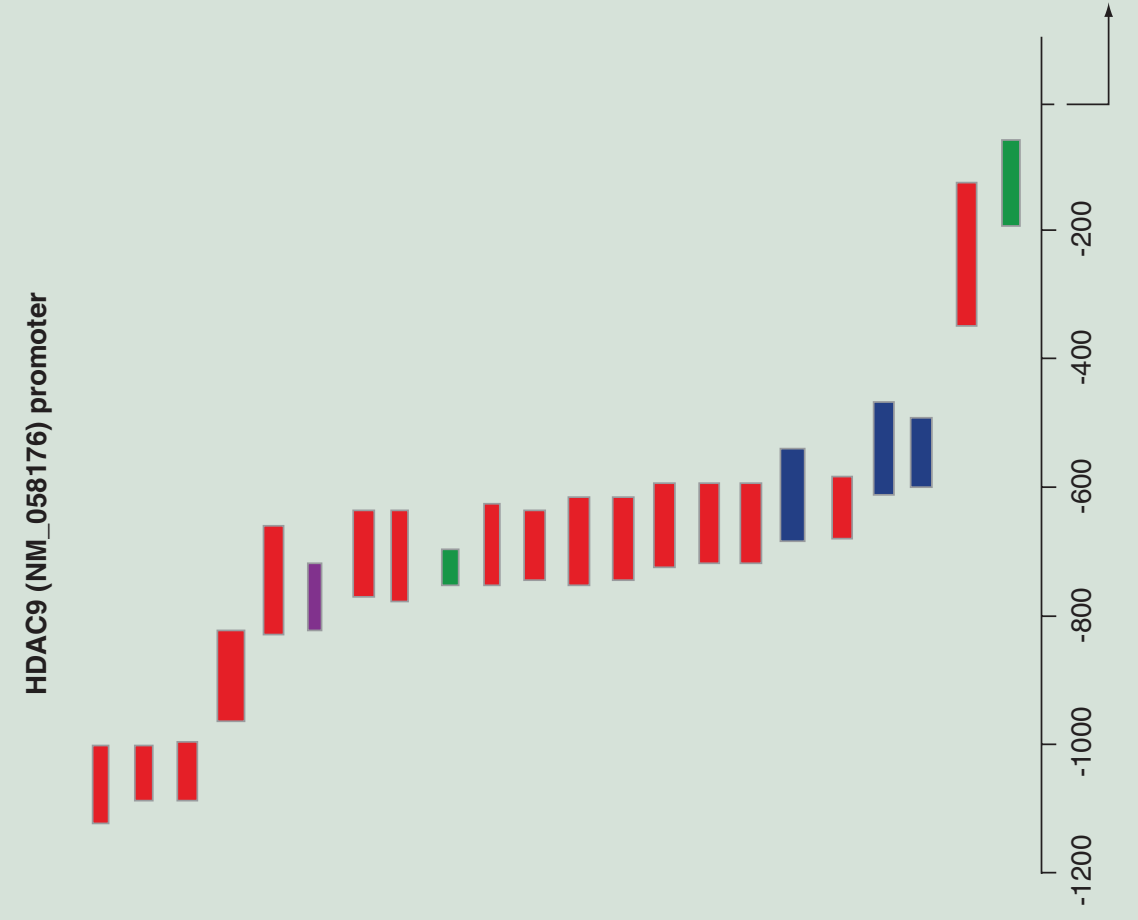

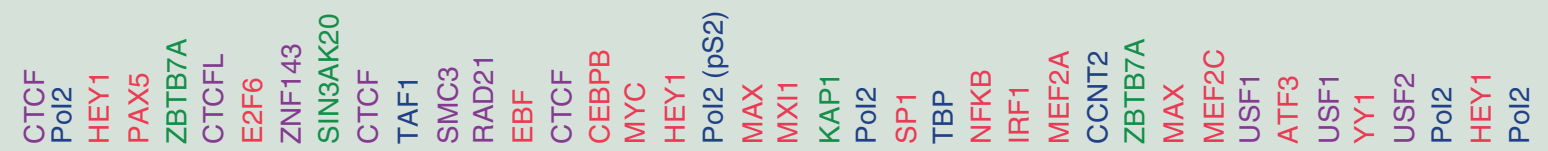

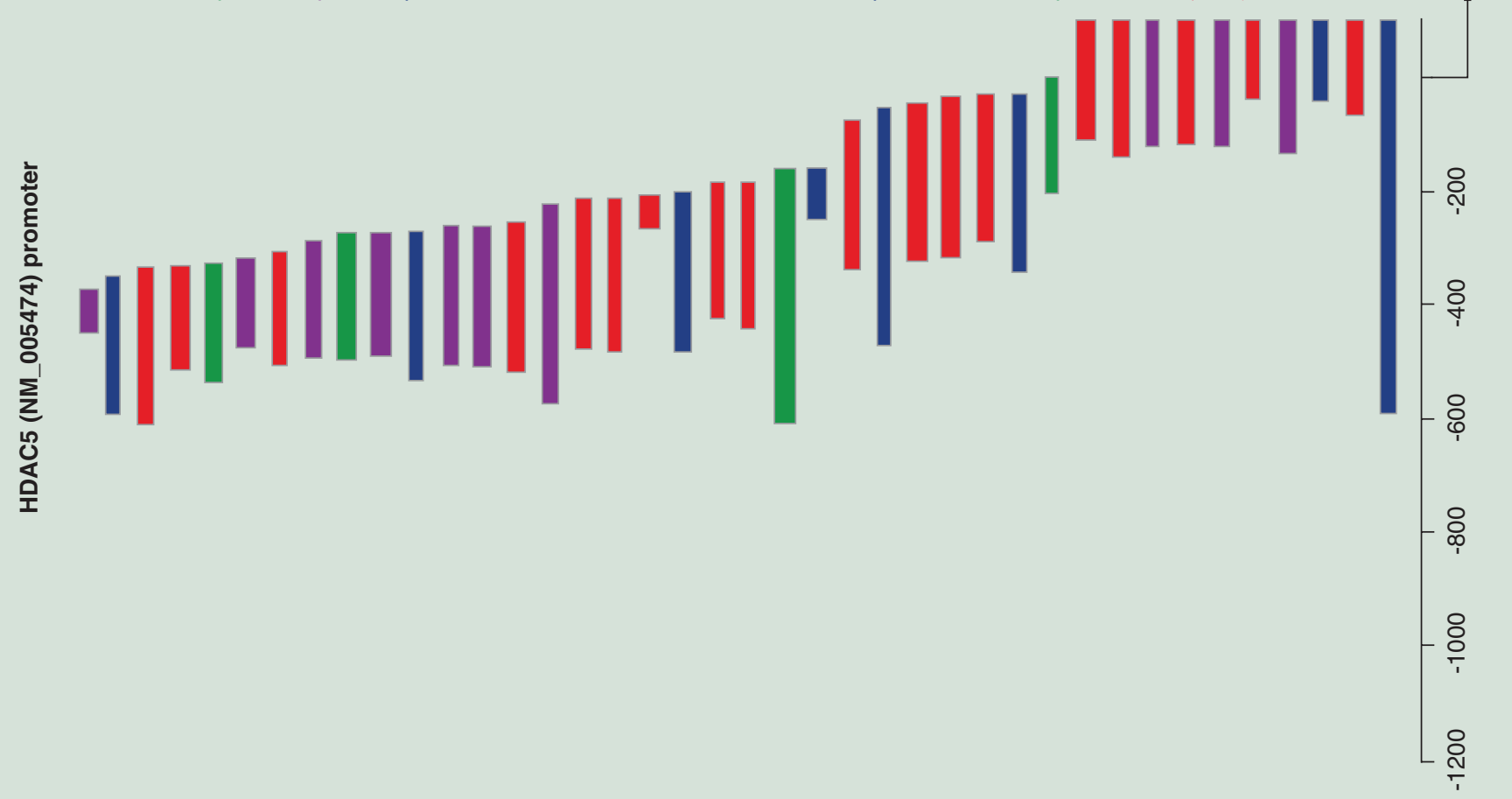


Figure 2. Available ChIP-Seq data from eight different cells lines were analyzed for the binding to the proximal promoters (-1/-1000) of HDAC5 and HDAC9 (see facing page). Transcription factors (red), epigenetic modifiers (green), basic transcriptional machinery (blue), architectural proteins (violet) and their relative region of binding are indicated.

the targeting of $H D A C 5$ and the subsequent activation of RUNX2 [51]. miR-2861 also targets the 3'UTR of HDAC5 in hamster ovary $\mathrm{CHO}$ cells [52]; by decreasing HDAC5 levels, miR-2861 enhances the productivity of these cells that are frequently used as cell factories for the production of recombinant proteins, feeding the industrial and economic interest around this miRNA.

Very recent is the finding that HDAC9 dysregulation may be involved in the age-related bone loss [53]. In particular, old mice are characterized by an increase of miR-188 levels in bone marrow stromal cells. miR-188 targets HDAC9 and RICTOR expression and the silencing of these two genes seems to be sufficient to regulate the switch between osteogenesis and adipogenesis in bone marrow stromal cells [53].

\section{Control of the mRNA stability: miRNAs targeting class Ila HDACs in cancer}

Interestingly, most of the miRNAs that target the 3'UTR of class IIa HDACs are repressed in certain cancers and display tumor suppressive traits. This observation is in agreement with the reported oncogenic properties of class IIa HDACs [23,26]. miR-1 is repressed in several cancers, such as lung cancer [54], hepatocellular carcinoma (HCC) [55] and chordoma [56]. In these contexts its repression is associated to the upregulation of FoxP1, MET and HDAC4 [54,55]. A similar upregulation of HDAC4 was correlated, in several HCC samples, with a reduced expression of miR-22 [57]. HDAC4 is targeted also by miR-140. In osteosarcoma and colon cancer cells this miRNA is associated with chemosensitivity [58].

Waldenström macroglobulinemia, a rare B cell lowgrade lymphoma, is characterized by a reduced expression of miR-9* [59]. This microRNA directly affects the levels of HDAC4 and HDAC5. Restoring of miR-9* levels provokes the downregulation of class IIa HDACs and increases p21/CDKN1A levels [60,61], thus limiting Waldenström macroglobulinemia cells proliferation [59]. An autoregulatory loop, as above described for miR-1, involves miR-200a and it is deregulated in HCC. miR-200a affects the stability of HDAC4 and, in a feedback manner, the deacetylase decreases miR200a transcription, by disturbing the binding of Sp1 to its promoter. Downregulation of miR-200a enhances the proliferation and migration of HCC cells, whereas its upregulation inhibits both the responses [62].

Similar correlations between HDAC4, microRNAs and tumors have been observed in breast cancer, where low levels of miR-125a-5p are bad prognos- tic markers and the majority of the tumor suppressive properties of miR-125a-5p depends on HDAC4 suppression [63].

In B cells, unlike the aforementioned cases, the upregulation of a miRNA (miR-155) that targets HDAC4 has proliferative effects [64]. These differences could reflect cell lineages specificities or the contribution of additional genes under the influence of miR-155.

In tongue squamous cell carcinoma, miR-140-5p can influence the expression of a gene cluster that includes ADAM10, LAMC1, PAX6 and HDAC7. These genes affect cell motility and could be responsible for the metastatic phenotype [65]. miR-34 can repress HDAC7 as well as $\mathrm{HDAC} 1$ expression. In breast cancer miR-34 is significantly downregulated, HDAC1 and HDAC7 levels are augmented and as a consequence HSP70 is deacetylated. The authors proposed that such modification confers resistance to chemotherapy-induced autophagic cell death [66].

A summary of the different microRNAs targeting class IIa HDACs is reported in Table 2.

\section{Proteolytic processing or massive degradation}

Selective proteolysis can influence class IIa HDACs activities. During apoptosis, HDAC4 and HDAC7 are cleaved, respectively, by caspase-3 [67] and caspase- 8 [68]. In both cases the cleavage products increase the apoptotic rate [67-69], but only for HDAC4 the amino-terminus generated-fragment is competent for MEF2 repression [67].

Another selective proteolytic processing was observed to modulate the hypertrophic response. In cardiomyocytes, protein kinase A (PKA) activation causes the cleavage of HDAC4 between residues 201 and 202, operated by an unidentified protease [70]. The generated amino-terminal fragment accumulates in the nucleus and it is competent for MEF2 repression but it is incompetent for SRF repression [70]. The binding site for PKA is present only in HDAC4 (aa 638-651), among all class IIa HDACs. The anti-hypertrophic effect of PKA is sufficient to antagonize the prohypertrophic actions of CaMKII, without affecting cardiomyocyte survival [70].

The ubiquitin-proteasome system (UPS) can also affect class IIa HDACs levels. Treatment of HEK293 cells with ALLN and MG132, two inhibitors not entirely proteasome-specific, provokes an increase in the levels HDAC4, HDAC5 and HDAC7 [71]. In the case of HDAC7, the authors suggested that 
Table 2. miRNAs targeting class Ila histone deacetylase.

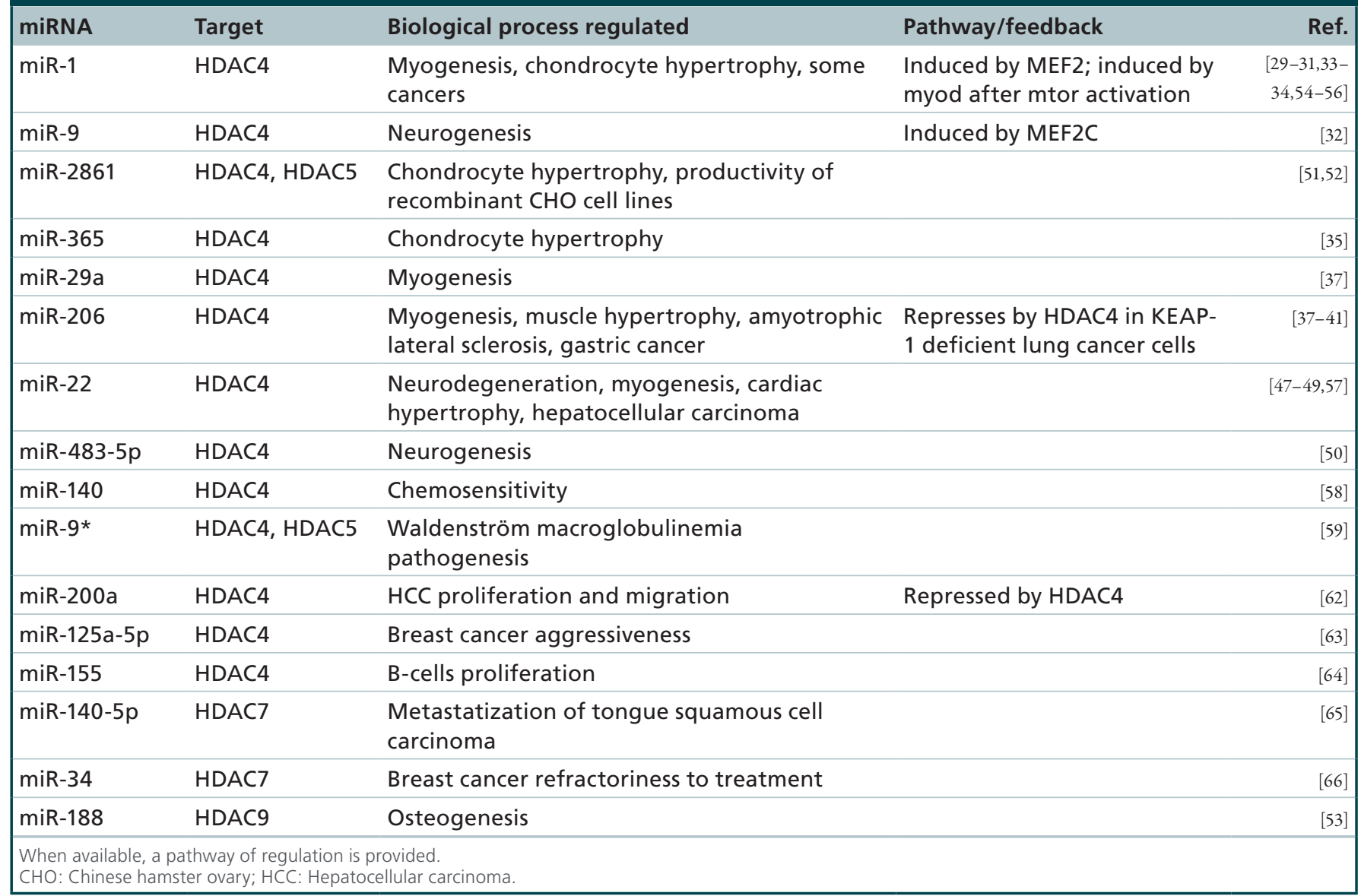

HDAC7 is degraded mainly in the cytoplasm after its phosphorylation-mediated export from the nucleus [71].

The UPS degradation of HDAC7 in the cytoplasm was recently confirmed during endochondral ossification [72]. Class IIa HDACs are negative modulators of endochondral ossification, at the stage of chondrocyte hypertrophy, by repressing the activity of RUNX2 and MEF2s $[18,73]$. HDAC7 is highly expressed in proliferating cells within the growth plate and its postnatal deletion increases the proliferation rate, because of $\beta$-catenin activation. During chondrocytes maturation, HDAC7 is exported into the cytoplasm where it is degraded by the UPS and liberates $\beta$-catenin [72].

The degradation of HDAC4 and HDAC5 was observed in vivo in muscles during fiber type conversion [74]. Contrary to HDAC7, the degradation of these class IIa HDACs takes place in the nucleus [74]. Nuclear degradation of class IIa HDACs was confirmed in untransformed cells exposed to serum starvation [75]. In this case GSK3 $\beta$ phosphorylates HDAC4 on serine 298 and this phosphorylation acts as a priming event required for its ubiquitylation and nuclear degradation (Figure 3 and Table 3) [75].
Degradation of class IIa HDACs as an environment driven process was observed also in a rat osteoblastic cell line. Here, the stimulation of differentiation with parathyroid hormone (PTH) causes PKA-dependent phosphorylation of HDAC4 on serine 740, its export in the cytoplasm and the degradation through a system that is lysosomal dependent [76]. Another set of studies using mice knock-out for HDAC4 and HDAC5 indicates that during osteoclast differentiation, PTH signaling favors MEF2C-dependent transcription by inducing HDAC4 poly-ubiquitylation and degradation via the E3 ubiquitin ligase SMURF2 [77]. Whether these differences reflect a specific cellular context still needs to be investigated.

Class IIa HDACs can also be SUMOylated [11]. In particular, HDAC4 becomes SUMOylated on lysine 559 by the SUMO E3-ligase RanBP2 on the nucleopore complex, during nuclear import [11]. The SUMOylation increases the interaction of HDAC4 with HDAC3 and therefore its repressive capability [11]. Also HDAC5 and HDAC9, but not HDAC7, are SUMOylated respectively on lysines 605 and 549 [11]. The lack of HDAC7 SUMOylation is probably due to the absence of the glutamine rich region [78]. 
Class IIa HDACs are not merely targets of SUMO E3-ligases, but several evidences indicate that they could promote the SUMOylation of some partners. They are involved in the activation of Ubc9, the SUMO E2-ligase [79]. In this manner, class IIa HDACs promote the SUMOylation of MEF2s [79], of promyelocytic leukemia protein [80] and of the nuclear receptors LXR $\alpha / \mathrm{NR} 1 \mathrm{H} 3$ and LXR $\beta / \mathrm{NR} 1 \mathrm{H} 2$ [81]. In the last example, SUMOylation stimulates the binding of the nuclear receptors to STAT1 and thus the inhibition of an inflammatory response [81].

\section{In \& out from the nucleus}

The control of the nuclear/cytoplasm shuttling is a widespread strategy to influence class IIa HDACs activities. This regulation provides evident advantages in terms of reaction time. A redistribution of class IIa between the two compartments is a quick response that allows an immediate and reversible adaptation of the cells to the new environmental conditions [82-84]. Once dephosphorylated and nuclear, class IIa HDACs may associate with HDAC3 and N-CoR/SMRT, forming the enzymatically active multiprotein complex capable of driving epigenetic changes [85].

Since class IIa HDACs exert their function mainly in the nucleus, a cytoplasmic accumulation is generally considered as a negative regulation [23,86]. For example, the nuclear localization prevails in undifferentiated cells, whereas differentiated cells prevalently accumulate class IIa in the cytosol [87].

By controlling the availability of the NLS to bind importin- $\alpha$ and of the NES to bind CRM1, cells modulate class IIa HDACs protein localization. Phosphorylation is the PTM used to modulate these bindings and hence, to control class IIa subcellular localization (Figure 3 \& Table 3). CaMKII was initially discovered as the kinase that elicits the nuclear export [86]. Today we know that various kinases are involved in such task. The supervisors of the opposite action (dephosphorylation and nuclear import) were discovered much later, associated to the activity of the phosphatases PP1 and PP2A [84,88-89].

The control is operated through the phosphorylation of at least three (four in HDAC7) serine residues conserved among HDAC4, 5, 7 and 9 (HDAC4: Ser 246, 467, 632; HDAC5: Ser 259, 497, 661; HDAC7: Ser 155, 181, 321, 446; HDAC9: Ser 220, 451, 611); these phosphorylation events facilitate the binding by dimers of 14-3-3 chaperones [90,91].

Binding of 14-3-3 proteins could either mask the NLS, thus preventing the nuclear import [82,83], or unmask the NES and thus promoting the direct interaction with CRM1 [89], or both conditions (although direct evidences of an interaction between HDAC4 and CRM1 are not available). It is still an open question whether the interaction between 14-3-3 proteins and class IIa HDACs occurs in the nucleus, in the cytoplasm or in both compartments. The interaction with 14-3-3 proteins would induce a conformational change in the deacetylases, which would make them able to expose the carboxy-terminal fragment containing the NES to CRM1 [86]. A work by Nishino et al. proposed that instead, the 14-3-3 proteins act primarily by slowing down the nuclear import of the deacetylases, in particular of HDAC4 [83].

In cardiomyocytes, HDAC4 is phosphorylated by the splicing isoforms, b and c, of CaMKII $\delta$ and this finding demonstrates that the phosphorylation of HDAC 4 could occur both in the nucleus (isoform b) and in the cytoplasm (isoform c). In the first case the export is favored whereas, in the second, the nuclear import is prevented [92].

Different class IIa HDACs members evidence different propensity to accumulate into the nucleus. For example, in transformed fibroblasts HDAC5 is almost nuclear, while HDAC4 is largely cytoplasmic or present in both compartments [93,94]. This observation indicates that within the same cell the two HDACs undergo different cycle of phosphorylation/dephosphorylation.

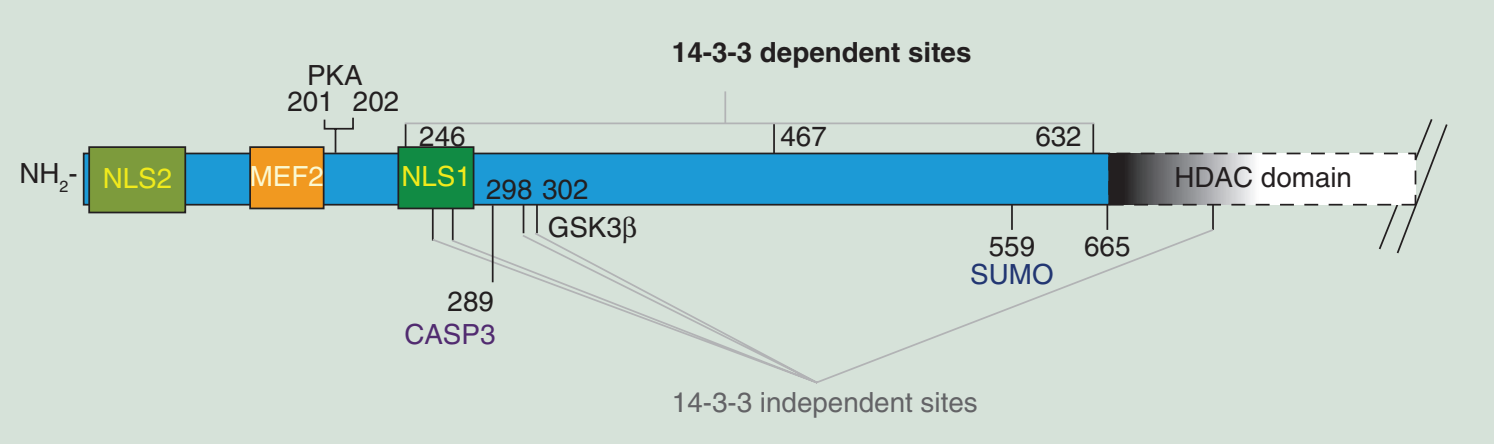

Figure 3. Schematic representation of class Ila histone deacetylases amino-terminal region highlighting the principal domains and the 'canonical' and 'not canonical' 14-3-3 phosphorylation sites see also Table 3. As prototype of class Ila we selected HDAC4. 
Table 3. Main kinases involved in class Ila histone deacetylases phosphorylation.

\begin{tabular}{|lllll|}
\hline Kinase & HDAC4 & HDAC5 & HDAC7 & HDAC9 \\
CaMKI, IV & 246,467 & 259,497 & $155,181,321$ & 220,451 \\
\hline CaMKII & 467,632 & - & - & - \\
PKD1 & $246,467,632$ & $259,497,661$ & $155,181,321,446$ & $220,451,611(?)$ \\
\hline C-TAK1, EMK & 246 & 259 & 155 & $220(?)$ \\
\hline AMPK & $246,467(?)$ & 259,497 & $?$ & $?$ \\
\hline SIK1 & 246,467 & 259,497 & $155,321(?)$ & $220,451(?)$ \\
SIK2 & $246,467,632$ & $259,497,661$ & $155,321,446$ & $220,451,611(?)$ \\
\hline PKA & $265,266,740$ & 278,279 & - & 243 \\
\hline DIRK1B & 266 & 276 & & 240 \\
\hline GSK3 $\beta$ & 298,302 & 278 & & 242 \\
\hline AURKB & 265 & 279 & - & - \\
\hline CDK5 & - & & \\
\hline $\begin{array}{l}\text { Phosphorylation together } \text { with the residues targeted. } \\
\text { ?: Not defined. }\end{array}$ & & & \\
\hline
\end{tabular}

In this review the discussion is focused on three families of kinases.

\section{Calcium-regulated kinases}

The kinases responsive to calcium are historically associated to HDACs nuclear export. CaMKI and IV phosphorylate all family members and show preference for residues 246 and 467 (in HDAC4 and the corresponding aa in other deacetylases), while CaMKII preferentially phosphorylates serines 467 and 632 of HDAC4 [87,95]. CaMKII phosphorylates and exports directly only HDAC4, because only HDAC4 has a CaMKII-specific docking site, centered on Arg601 [95]. However, since HDAC4 can form heterodimers with HDAC5 and 9, but not with HDAC7 [96], the association between HDAC 4 and HDAC5/9 renders such proteins responsive to CaMKII. In particular HDAC4 interacts strongly with HDAC5 through the glutaminerich region [96]. The lack of this region in HDAC7 explains its inability to interact with HDAC4 $[78,96]$.

The calcium-mediated export of class IIa HDACs is involved in the regulation of many physiological processes, such as myogenesis, hypertrophy and neuronal survival [97-99]. In general, the prosurvival effect associated to HDAC4 nuclear export depends on the activation of a MEF2-transcriptional response [98].

In cardiomyocytes, the pro-hypertrophic stimuli can be transduced by cAMP via the exchange protein directly activated by cAMP sensor, which activates PLC, H-Ras and CAMKII. This pathway culminates in the cytoplasmic accumulation of HDAC4 [100]. Exchange protein directly activated by cAMP is a guanosine nucleotide exchange factor for the Rap small GTPase. In particular PLC, and the subsequent sig- naling cascade of the inositol 1,3,5 triphosphate causes a release of calcium in the cytoplasm that determines the export of HDAC4 via CaMKII, followed by the activation of MEF2-dependant transcription [100].

For further and more detailed reviews on calcium-mediated class IIa regulation see [5,90,91].

\section{PKD}

PKD, a serine/threonine kinase activated by PKC, was associated to class IIa HDACs export during lymphocyte maturation and thymic selection $[101,102]$. B-lymphocyte activation, following BCR (B cell receptor) engagement is accompanied by the stimulation of PKD1 and PKD3, which in turn phosphorylate HDAC5 and HDAC7 on classical 14-3-3 sites. As a consequence, HDAC5 and HDAC7 accumulate in the cytoplasm and chromatin relaxation can occur [102], thus switching on the MEF2 transcriptional program [103]. Since the two kinases are redundant, in order to abrogate the export of HDAC5, a double knock-out of PDK1 and PDK3 is required.

PKD1 is also a regulator of T-lymphocyte thymic selection [104]. In 'resting' double positive $\mathrm{CD}^{+} \mathrm{CD}^{+}$ thymocytes, nuclear HDAC7 maintains switched off the MEF2 genetic program and in particular the transcription of the proapoptotic nuclear receptor Nur77/NR4A1, the main responsible for the negative selection. In response to T-cell receptor engagement, PKD1 becomes active and phosphorylates HDAC7, which is exported into the cytoplasm. This export determines the activation of MEF2s and the derepression of NR4A1 that promotes cell death [101,104].

PKD is also an important inducer of cardiac hypertrophy via phosphorylation and export of HDAC5 and 
the relative derepression of MEF2 [105]. Mice with a cardiac-specific deletion of PKD1 show diminished hypertrophy in response to pressure overload or chronic adrenergic and angiotensin II signaling. Several selective inhibitors of PKD are now under clinical studies for the treatment of malignant cardiac hypertrophy [105]. PKD1 seems to be an inducer of hypertrophy also in VSMCs. Here, treatment with the hypertrophic inducer angiotensin II stimulates the phosphorylation of HDAC5 via PKD1 [106]. Similarly, angiotensin II through PKD1 triggers HDAC4 (serines 246/632), HDAC5 (serines 259/498) and HDAC7 (serine 155) phosphorylation in intestinal epithelium [107].

Finally, exogenous expression of PKD1 in type II skeletal muscle fibers promotes the phosphorylation and the nuclear export of HDAC4 and HDAC5, the subsequent activation of MEF2 TFs and the fibersswitch from fast/glycolytic into red oxidative (slowtwitch type I) [108], in accordance to the phenotype observed in transgenic MEF2C-VP16 mice [74].

\section{LKB1-ARK family}

The tumor suppressor kinase LKB1 regulates different downstream kinases, which belong to the ARK family (AMP-related kinases) and includes AMP-activated protein kinase (AMPK), microtubule affinity regulating kinases (MARKs), SNF-related kinases (SNRKs), NUAKs (NUAK family, SNF1-like kinases), BR serine/threonine kinases and salt inducible kinases (SIKs) [109].

Early studies reported that MARK kinases phosphorylate class IIa HDACs constitutively on a conserved residue (S159 in HDAC7, S246 in HDAC4, S259 in HDAC5). This base-line phosphorylation facilitates the subsequent signal-dependent phosphorylation by other kinases of the remaining residues required for 14-3-3 binding [110]. MARK/PAR-1 kinases are involved in the determination of polarity. During early embryogenesis they control gastrula polarization in Drosophila [11] and the first asymmetric division in Caenorhabditis elegans [112]. Scenarios where the nuclear or cytoplasmic activities of class IIa HDACs are still unexplored.

\section{The AMPK}

The AMPK is activated under conditions of metabolic stress and ATP depletion [109]. Some evidences correlate AMPK activity to HDAC4 and HDAC5 cytosolic accumulation [113,114]. In skeletal muscle, the stress induced by physical exercise is sufficient to trigger the export of HDAC4 and 5 and this relocalization correlates with the activation of AMPK and CaMKII [115]. In myotubes the AMPK agonist AICAR (5-aminoimidazole-4-carboxamide-1-beta-D-ribofuranoside) triggers HDAC5 phosphorylation at S259 and S498 and association with 14-3-3 isoforms. HDAC5 phosphorylation determines its detachment from the GLUT4 promoter and the concomitant increase in GLUT4 expression [116]. This metabolic regulation can be supervised also by other kinases such as PKDs [117].

\section{The SIK subfamily}

The SIK-subfamily is composed of three isoforms: SIK1, SIK2 and SIK3, conserved from C. elegans to humans. SIK1 expression is highly induced in adrenal glands of high-salt diet-fed rats, whereas SIK2 is highly expressed in adipose tissue. By contrast, SIK3 is ubiquitously expressed [118]. Several studies have reported correlations between SIKs activities, class IIa HDACs relocalization and transcriptional changes [119-121].

The SIK-class IIa HDAC axis is a critical component of the adaptation to fasting in liver [22]. After feeding, the release of insulin stimulates in liver and skeletal muscle, the synthesis of glycogen and in adipocytes the storage of energy reserves. These responses involve the engagement of Akt and culminate in the phosphorylation-mediated inactivation of PGC-1 $\alpha$ and FOXO1/3 [122]. The FOXO TFs are also negatively regulated by acetylation, which promotes their export into the cytoplasm. Here, the SIK-class IIa HDACs axis becomes protagonist.

In mouse hepatocytes, in response to insulin SIK2 mediates phosphorylation, 14-3-3 binding and cytosolic accumulation of HDAC4. Accordingly, the fasting hormone glucagon, through the PKA-mediated inhibition of SIK2 favors dephosphorylation and nuclear accumulation of HDAC4 [123].

Inhibition of LKB1/AR kinases is followed by the nuclear relocalization of class IIa HDACs, a key-event in order to promote the activation of the gluconeogenesis in the liver. Nuclear class IIa HDACs associated with HDAC3 in order to deacetylate and activate FOXO1/3. Then, FOXO1/3 stimulate the transcription of key enzymes for the gluconeogenesis [22].

In the adipose tissue, modulation of SIK2 influences HDAC4 phosphorylation through a multiprotein complex, which comprises also CREB-regulated transcription co-activator 2 and 3, as well as PP2A. This complex is under the supervision of PKA and is involved in the regulation of GLUT4 transcription and glucose uptake [124].

Recently, HDAC4 has been described as an immune-metabolic sensor. In particular under overnutrition, leptin reduces inflammatory gene expression via HDAC4 nuclear accumulation [125]. PKA-dependant inhibition of SIKs (following AMPc increase) represents the operative arm that allows the HDAC4dependent repression of NF- $\kappa \mathrm{B}$ activity and of proin- 
flammatory genes in M2 macrophages [125]. Because obesity promotes macrophage infiltration in white adipose tissues and liver, inflammation and subsequent insulin resistance, the negative effect of HDAC4 on NF- $\mathrm{kB}$ suggests for a protective role against obesity [125]. In agreement, HDAC4 variants have been associated with both body mass index and waist circumference [125] and its expression is downregulated in the fat from obese subjects [126].

The involvement of the HDACs-SIKs axis in the control of energy supply and metabolism seems to be conserved during evolution. In Drosophila HDAC4 nuclear accumulation in the fat body cells is supervised by the LKB1-SIK3 signaling, under the supervision of different dietary conditions. SIK3 controls lipid metabolism by limiting HDAC4-mediated FOXO activation and $A T G L / B m m$ transcription (adipose triglyceride lipase). In the control of lipid metabolism HDAC4 behaves as a lipolytic factor [127].

SIKs can monitor class IIa HDACs activities also independently from metabolism. In muscle cells, SIK1 phosphorylates class IIa HDACs thus promoting their export into the cytoplasm [119,128]. In this context, SIK1 could integrate cAMP signaling with the myogenic program [129]. In C. elegans the expression of chemoreceptors gene in certain chemosensory neurons is under the regulation of a SIK member (KIN29), which controls the localization of the class IIa HDAC counterpart (HDA-4). This control releases the repressive influence of HDA-4 on MEF2-target genes. In the circuit participate also the phosphatase Calcineurin, which instead promotes HDA-4 nuclear accumulation and chemoreceptors repression [130].

Finally, in a detailed study performed in HEK 293 cells, Walkinshaw et al. evaluated 13 kinases of the LKB1 family (MARK1, MARK2, MARK3/CTAK1, NUAK1/ARK5, NUAK2/SNARK, SNRK, NIM1) for the regulation of class IIa HDACs subcellular localization [94]. They demonstrated that only the ectopic expression of SIK2 or SIK3 but not of SIK1 causes a dramatic cytoplasmic relocalization of HDAC5 and HDAC9 and, at a lesser extent, of HDAC4 and HDAC7. For the cytoplasmic relocalization of class IIa HDACs the catalytic activity of SIK2 is required, while the catalytic activity of SIK3 is dispensable. Moreover, while SIK2 promotes the nuclear export through the phosphorylation of 14-3-3 consensus sites, SIK 3 is effective also on the Ser/Ala mutants in the 14-3-3 binding sites of HDAC4. This result proves that the SIK3-mediated export is both kinase activity and classical 14-3-3 binding sites independent [94]. Finally, while SIK2 causes the derepression of MEF2 and stimulates myogenesis in C2C12 cells, SIK3 is incompetent toward MEF2 activation [96].
Whether these differences reflect cell lineage specific features or others conditions is currently unknown.

The large number of studies on the LKB1-SIKsclass IIa HDACs axis underlines the extreme flexibility of the class IIa HDACs, and in particular of the nuclear/cytoplasmic regulation. By a simple operation (nuclear exit) it is possible to reset the transcriptional landscape of cells by both inducing MEF2-target genes and repressing FOXO-target genes.

\section{Phosphatases \& nuclear import}

More than 15 years ago it was demonstrated that Calyculin A, an inhibitor of the phosphatases PP1 and PP2A, promotes the nuclear export of HDAC4 and reduces its interaction with importin- $\alpha$ [82]. Several years later the contribution of the PP2A complex was proved. PP2A is able to bind the amino-terminal portion of class IIa HDACs, in correspondence of the NLS1 and to dephosphorylate these regulators $[84,88,89]$.

In HDAC 4 two residues have been proposed as targets of PP2A: serine 246 and 298. Serine 298 is also regulated by GSK3 $\beta$ and constitutes a signal for polyubiquitylation and degradation [75]. The PP2A-mediated dephosphorylation may therefore also protect HDAC4 from the UPS-mediated nuclear degradation.

The PTH-related peptide suppresses MEF2 and RUNX2 transcriptional activities and chondrocyte hypertrophy, via PP2A, which dephosphorylates HDAC4 at serine 246 [89]. PP2A is sufficient to trigger the import of HDAC4 and inhibition of PP2A, in $\mathrm{U} 2 \mathrm{OS}$ cells, causes the cytoplasmic relocalization of the deacetylase [89].

Ataxia telangiectasia is a complex syndrome characterized by neurodegeneration and epigenetic reprogramming and caused by mutations of the ATM kinase [131]. Since ATM phosphorylates and inhibits PP2A, ataxia telangiectasia is characterized by HDAC4 nuclear accumulation in neurons. Here, HDAC4 represses MEF2 and CREB thus inducing heterochromatinization and neurodegeneration [132].

The myosin phosphatase complex, consisting of PP1 $\beta$ and MYPT1, is necessary to repress NUR77. This complex is able to dephosphorylate HDAC7, thus stimulating its nuclear relocalization [94,133]. In smooth muscles, the myosin phosphatase dephosphorylates the myosin light chain, thus inducing muscle relaxation and it is inactivated following the phosphorylation of MYPT1 on threonine 696 [134]. Kinases regulated by the GTPase RhoA are able to phosphorylate and inhibit myosin phosphatase on this residue [134] and they are also responsible for the nuclear export of HDAC5 [134]. It is therefore possible that RhoA activation could neutralize MYPT1, thus 
resulting in the hyperphosphorylation and export of HDAC5 [134].

PP1 $\alpha$ is another phosphatase capable of mediating HDAC4 nuclear import. Its action can overcome the antagonist activating effect of calcineurin on MEF2 [135].

The regulation of class IIa HDACs phosphorylation is a complex issue, not limited to 14-3-3 binding sites but involving additional residues. In the case of HDAC5, at least 17 phosphorylation sites have been characterized, 13 of which do not encompass the consensus for 14-3-3 proteins [136]. In particular, the phosphorylation of serine 279 is essential to induce the nuclear import of the protein. This residue is conserved among all class IIa HDACs, with the exception of HDAC7 [136]. Curiously, this residue was previously characterized as an Mirk/DirkB target, but in this case its phosphorylation causes the nuclear export of class IIa HDACs [137]. Additional kinases responsible for HDAC5 Ser279 phosphorylation have been identified in PKA and CDK5 [138,139]. PKA retains HDAC5 in the nucleus by interfering with 14-3-3 binding, thus causing suppression of MEF2-dependant cardiac fetal gene expression and cardiomyocyte hypertrophy [138]. On the opposite, CDK5 promotes HDAC5 nuclear export in neurons. In this context, cocaine administration activates PP2A, which dephosphorylates Ser279 and determines the nuclear import of HDAC5 [139]. In the nucleus HDAC5 can repress genes regulated by cocaine [139].

The opposite influence on HDAC5 subcellular localization by the phosphate group on serine 279 is puzzling. Additional substrates of these kinases could explain the paradox. It is evident that further studies are necessary to clarify this point.

\section{4-3-3 independent regulation of subcellular localization}

There are some indications about a 14-3-3-independent control of class IIa nuclear/cytoplasmic shuttling. An example concerns the import regulated by TRX1 (thioredoxin 1) in cardiomyocytes [140]. TRX1 is able to recruit HDAC4 in the nucleus and to inhibit the activity of some pro-hypertrophic factors such as MEF2 and NFAT [140]. This adjustment of HDAC4 'shuttling' is tissue-specific and mediated through the control of the redox state of two cysteines [140].

Cardiac hypertrophy is characterized by an increase in intracellular reactive oxygen species (ROS) [90]. Under oxidizing conditions, a disulfide bridge between the cysteines 667 and 669 is formed [140]. Cysteine 667 lies in the binding site for the 'structural' zinc ion $[141,142]$. In a reducing environment, cysteines 667 and 669 and the zinc ion bound to its binding site leads to a protein folding that brings the structural zinc-binding domain in contact with the NES. In this manner the CRM1 binding site is masked and the nuclear export is blocked. In the presence of oxidants, cysteines 667 and 669 are oxidized, the zinc is no longer coordinated, the NES is exposed to CRM1 and the protein is exported into the cytoplasm [140]. TRX1 is able to attenuate cardiac hypertrophy in part by restoring the cytoplasmic reducing environment. Furthermore, after the binding to TBP-2, TRX1 reduces DnaJB5 (Hsp40), which in this state can interact with HDAC4. Next the complex TRX1TBP-DnaJB5 reduces the disulfide bridge 667-669 of HDAC4. This intervention is sufficient for determining the nuclear accumulation of HDAC4, in spite of its phosphorylation status $[140,143]$. Similarly to HDAC4, also HDAC5 has been recently described as a redox-sensor in adult heart [144].

As discussed above, the AMPc through PKA owns different options to influence class IIa HDACs subcellular localization. An additional opportunity consists in the PKA-promoted (clearly indirect) dephosphorylation of serines 265/266 in HDAC4, independently from the above-described action on SIK2. These residues are conserved in HDAC5 (278/279) and in HDAC9 (242/243) and lie within the NLS [94]. Their dephosphorylation favors class IIa nuclear accumulation and the subsequent repression of MEF2D and myogenesis [138]. However, since PKA phosphorylates also MEF2D [145], it is difficult to discriminate between the repressive activity of PKA due to the direct phosphorylation of MEF2s or the nuclear import of a class IIa HDACs.

During the cell cycle, HDAC4, HDAC5 and HDAC9, but not HDAC7 can be phosphorylated by Aurora B kinase, respectively on Ser265, Ser278 and Ser242. These phosphorylations allow the relocalization of the deacetylases at the mitotic midzone during late anaphase, and in the midbody during cytokinesis [146]. This phosphorylation-dependent relocalization abolishes the interaction with the NCoR complex, thus limiting part of class IIa deacetylase activity [146].

Overall, the regulation of class IIa HDACs functions during the cell cycle is largely unexplored, although it could provide important hints on the relationships between this gene family and the control of proliferation.

\section{Conclusion}

In this review we have discussed about the multiple options that cells have evolved to control class IIa HDACs. Although the phosphorylation-dependent control of nuclear cytoplasmic shuttling takes a chief 
role, additional opportunities are emerging such as the control of transcription and translation. In summary, cells dispose of different operating layers to influence class IIa HDACs activities. This complexity reflects the astonishing number of biological processes under the supervision of this gene family. It seems that each control is sculptured under the needs of specific conditions.

\section{Future perspective}

Although studies on class IIa HDACs are intense, there are several important questions, which still need to be answered, for example, cytoplasmic versus nuclear functions, redundancy, compensatory regulative circuits, transcriptional repression and activation, histones versus transcription factors modifications and many others issues. Investigation on class IIa HDACs will certainly proceed in the next future and addi- tional pieces to this intricate puzzle will be step by step added.

Financial \& competing interests disclosure

Research activities in the laboratory of C Brancolini are supported by AIRC (IG-10437) and MIUR Progetto PRIN (Progetto 2010W4J4RM_002). The authors have no other relevant affiliations or financial involvement with any organization or entity with a financial interest in or financial conflict with the subject matter or materials discussed in the manuscript apart from those disclosed.

No writing assistance was utilized in the production of this manuscript.

\section{Open access}

This work is licensed under the Attribution-NonCommercialNoDerivatives 4.0 Unported License. To view a copy of this license, visit http://creativecommons.org/licenses/by-nc-nd/4.0/

\section{Executive summary}

- Class Ila histone deacetylases (HDACs) are exposed to multilayered levels of controls, reflecting the multiple biological responses under their supervision.

Transcriptional control

- Few data are available about the transcriptional machinery responsible for class Ila HDACs expression.

- Interrogation of ENCODE database indicates that several transcription factors (TFs) including oncogenes and cell cycle regulators bind the proximal promoter of class Ila HDACs.

- HDAC4 and HDAC7 proximal promoters are exposed to intense TFs and epigenetic modifiers binding.

Translational control

- Different microRNAs can influence class Ila HDACs translation.

- In some cases, class Ila HDACs are part of feedback circuits controlling the transcription of their own regulative microRNA.

- Further studies are necessary to clarify the effective contribution of class Ila HDACs to the biological role of the specific microRNAs.

Proteolytic control

- Class Ila HDACs levels can be regulated by the ubiquitin-proteasome system and further studies are necessary to define the E3 ligases involved.

- Specific proteolytic cleavages can also influence class Ila HDACs activities in a member specific fashion.

- Although the involvement of caspases during apoptosis is well known, additional proteases operating in different circumstances may exist.

Phosphorylation, 14-3-3 binding \& subcellular localization

- The most common and widespread mechanism to influence class Ila HDACs activities.

- Several environmental conditions and signaling pathways use this post-translational modification for influencing class Ila HDACs.

- A fast response that allows an immediate and reversible adaptation of the cells to the new environmental conditions.

14-3-3 independent regulation of subcellular localization

- Less characterized, nevertheless experimental evidences indicate that 14-3-3 independent control of class Ila HDACs does exist.

- Up to now the best characterized mechanism involves the redox control of a pair of cysteine residues lied in the deacetylase domain of HDAC4.

Future perspective

- Future studies on class Ila HDACs will certainly provide additional key information about this important gene family. The mysterious change in the catalytic site occurred with the evolution of vertebrates is a challenging and fascinating question. Undoubtedly, the plethora of biological responses supervised by class Ila HDACs guarantees that, thanks to these studies, new tools and opportunities for the treatment of different human diseases will be established. 


\section{References}

Papers of special note have been highlighted as:

- of interest; $\bullet \bullet$ of considerable interest

1 Choudhary C, Kumar C, Gnad F et al. Lysine acetylation targets protein complexes and co-regulates major cellular functions. Science 325(5942), 834-840 (2009).

2 Yang X-J, Seto E. The Rpd3/Hda1 family of lysine deacetylases: from bacteria and yeast to mice and men. Nat. Rev. Mol. Cell Biol. 9(3), 206-218 (2008).

3 Clocchiatti A, Di Giorgio E, Demarchi F, Brancolini C. Beside the MEF2 axis: unconventional functions of HDAC4. Cell. Signal. 25(1), 269-276 (2013).

4 Clocchiatti A, Florean C, Brancolini C. Class IIa HDACs: from important roles in differentiation to possible implications in tumourigenesis. J. Cell. Mol. Med. 15(9), 1833-1846 (2011).

5 Parra M. Class IIa HDACs - new insights into their functions in physiology and pathology. FEBS J. 282(9), 1736-44 (2015).

6 Thierry-Mieg D, Thierry-Mieg J. AceView: a comprehensive cDNA-supported gene and transcripts annotation. Genome Biol. 7(Suppl. 1), S12.1-S14.1 (2006).

7 Zhou B, Margariti A, Zeng L et al. Splicing of histone deacetylase 7 modulates smooth muscle cell proliferation and neointima formation through nuclear $\beta$-catenin translocation. Arterioscler. Thromb. Vasc. Biol. 31(11), 2676-2684 (2011).

8 Margariti A, Xiao Q, Zampetaki A et al. Splicing of HDAC7 modulates the SRF-myocardin complex during stem-cell differentiation towards smooth muscle cells. J. Cell Sci. 122(Pt 4), 460-470 (2009).

9 Petrie K, Guidez F, Howell L et al. The histone deacetylase 9 gene encodes multiple protein isoforms. J. Biol. Chem. 278(18), 16059-16072 (2003).

10 Zhang CL, McKinsey TA, Olson EN. The transcriptional corepressor MITR is a signal-responsive inhibitor of myogenesis. Proc. Natl Acad. Sci. USA 98(13), 7354-7359 (2001).

11 Kirsh O, Seeler JS, Pichler A et al. The SUMO E3 ligase RanBP2 promotes modification of the HDAC4 deacetylase. EMBO J. 21(11), 2682-2691 (2002).

12 Liu F, Pore N, Kim M et al. Regulation of histone deacetylase 4 expression by the SP family of transcription factors. Mol. Biol. Cell. 17(2), 585-597 (2006).

13 Sleiman SF, Langley BC, Basso M et al. Mithramycin is a gene-selective $\mathrm{Sp} 1$ inhibitor that identifies a biological intersection between cancer and neurodegeneration. J. Neurosci. 31(18), 6858-6870 (2011).

14 Vallianou NG, Evangelopoulos A, Schizas N, Kazazis C. Potential anticancer properties and mechanisms of action of curcumin. Anticancer Res. 35(2), 645-51 (2015).

15 Zhang L, Jin M, Margariti A et al. Sp1-dependent activation of HDAC7 is required for platelet-derived growth factor-BBinduced smooth muscle cell differentiation from stem cells. J. Biol. Chem. 285(49), 38463-72 (2010).

16 Zhou B, Margariti A, Zeng L et al. Splicing of histone deacetylase 7 modulates smooth muscle cell proliferation and neointima formation through nuclear -catenin translocation. Arterioscler. Thromb. Vasc. Biol. 31(11), 2676-2684 (2011).

17 Tang H, Macpherson P, Marvin M et al. A histone deacetylase 4/myogenin positive feedback loop coordinates denervation-dependent gene induction and suppression. Mol. Biol. Cell. 20(4), 1120-1131 (2009).

18 Vega RB, Matsuda K, Oh J et al. Histone deacetylase 4 controls chondrocyte hypertrophy during skeletogenesis. Cell 119, 555-566 (2004).

19 Barneda-Zahonero B, Román-González L, Collazo O et al. HDAC7 Is a repressor of myeloid genes whose downregulation is required for transdifferentiation of pre-B cells into macrophages. PLoS Genet. 9(5), e1003503 (2013).

20 Addis RC, Prasad MK, Yochem RL et al. OCT3/4 regulates transcription of histone deacetylase 4 (Hdac 4$)$ in mouse embryonic stem cells. J. Cell. Biochem. 111, 391-401 (2010).

21 Haberland M, Arnold MA, McAnally J, Phan D, Kim Y, Olson EN. Regulation of HDAC9 gene expression by MEF2 establishes a negative-feedback loop in the transcriptional circuitry of muscle differentiation. Mol. Cell. Biol. 27(2), 518-525 (2007).

22 Mihaylova MM, Vasquez DS, Ravnskjaer K et al. Class IIa histone deacetylases are hormone-activated regulators of FOXO and mammalian glucose homeostasis. Cell 145, 607-621 (2011).

23 Clocchiatti A, Di Giorgio E, Ingrao S, Meyer-Almes F-J, Tripodo C, Brancolini C. Class IIa HDACs repressive activities on MEF2-depedent transcription are associated with poor prognosis of $\mathrm{ER}^{+}$breast tumors. FASEB J. 27, 942-954 (2013).

24 Clocchiatti A, Di Giorgio E, Viviani G et al. The MEF2HDAC axis controls proliferation of mammary epithelial cells and acini formation in vitro. J. Cell Sci. 128(21), 3961-3976 (2015).

25 Rosenbloom KR, Dreszer TR, Long JC et al. ENCODE whole-genome data in the UCSC genome browser: update 2012. Nucleic Acids Res. 40, D912-D917 (2012).

26 Di Giorgio E, Clocchiatti A, Piccinin S et al. MEF2 is a converging hub for histone deacetylase 4 and phosphatidylinositol 3-kinase/Akt-induced transformation. Mol. Cell. Biol. 33, 4473-91 (2013).

27 Zhu J, Shimizu E, Zhang X, Partridge NC, Qin L. EGFR signaling suppresses osteoblast differentiation and inhibits expression of master osteoblastic transcription factors Runx2 and Osterix. J. Cell. Biochem. 112(7), 1749-1760 (2011).

28 Vella P, Barozzi I, Cuomo A, Bonaldi T, Pasini D. Yin Yang 1 extends the Myc-related transcription factors network in embryonic stem cells. Nucleic Acids Res. 40 (8), 3403-3418 (2012).

29 Chen J-F, Mandel EM, Thomson JM et al. The role of microRNA-1 and microRNA-133 in skeletal muscle proliferation and differentiation. Nat. Genet. 38, 228-233 (2006).

30 Liu N, Williams AH, Kim Y et al. An intragenic MEF2dependent enhancer directs muscle-specific expression of microRNAs 1 and 133. Proc. Natl Acad. Sci. USA 104, 20844-20849 (2007). 
31 Ikeda S, He A, Kong SW et al. MicroRNA-1 negatively regulates expression of the hypertrophy-associated calmodulin and Mef2a genes. Mol. Cell. Biol. 29, 2193-2204 (2009).

32 Davila JL, Goff LA, Ricupero CL et al. A positive feedback mechanism that regulates expression of miR-9 during neurogenesis. PLoS ONE 9(4), e94348 (2014).

33 Sun Y, Ge Y, Drnevich J, Zhao Y, Band M, Chen J. Mammalian target of rapamycin regulates miRNA-1 and follistatin in skeletal myogenesis. J. Cell Biol. 189, 1157-1169 (2010).

34 Li P, Wei X, Guan Y, Chen Q, Zhao T, Sun C, Wei L. MicroRNA-1 regulates chondrocyte phenotype by repressing histone deacetylase 4 during growth plate development. FASEB J. 28( 9), 3930-3941 (2014).

35 Guan Y-J, Yang X, Wei L, Chen Q. MiR-365: a mechanosensitive microRNA stimulates chondrocyte differentiation through targeting histone deacetylase 4. FASEB J. 25, 4457-4466 (2011).

36 Li Z, Hassan MQ, Jafferji M et al. Biological functions of miR-29b contribute to positive regulation of osteoblast differentiation. J. Biol. Chem. 284(23), 15676-15684 (2009).

37 Winbanks CE, Wang B, Beyer C et al. TGF-b regulates miR-206 and miR-29 to control myogenic differentiation through regulation of HDAC4. J. Biol. Chem. 286, 13805-13814 (2011)

38 Williams AH, Valdez G, Moresi V et al. MicroRNA-206 delays ALS progression and promotes regeneration of neuromuscular synapses in mice. Science 326(5959), 1549-1554 (2009).

39 Valsecchi V, Boido M, De Amicis E, Piras A, Vercelli A. Expression of muscle-specific MiRNA 206 in the progression of disease in a murine SMA model. PLoS ONE 10(6), e0128560 (2015).

40 Ren J, Huang HJ, Gong Y et al. MicroRNA-206 suppresses gastric cancer cell growth and metastasis. Cell Biosci. 5(4), 26 (2014).

41 Singh A, Happel C, Manna SK et al. Transcription factor NRF2 regulates miR-1 and miR-206 to drive tumorigenesis. J. Clin. Invest. 123(7), 2921-2934 (2013).

42 Ago T, Liu T, Zhai P et al. A redox-dependent pathway for regulating class II HDACs and cardiac hypertrophy. Cell 133, 978-993 (2008).

43 Gagan J, Dey BK, Layer R, Yan Z, Dutta A. Notch3 and Mef2c proteins are mutually antagonistic via $\mathrm{Mkp} 1$ protein and miR-1/206 microRNAs in differentiating myoblasts. J. Biol. Chem. 287(48), 40360-40370 (2012).

44 Gruber AJ, Zavolan M. Modulation of epigenetic regulators and cell fate decisions by miRNAs. Epigenomics 5(6), 671-83 (2013).

45 Mielcarek M, Landles C, Weiss A et al. HDAC4 reduction: a novel therapeutic strategy to target cytoplasmic huntingtin and ameliorate neurodegeneration. PLoS Biol. 11(11), e1001717 (2013).

46 Mielcarek M, Toczek M, Smeets CJ et al. HDAC4-myogenin axis as an important marker of HD-related skeletal muscle atrophy. PLoS Genet. 11(3), e1005021 (2015).
47 Jovicic A, Zaldivar Jolissaint JF, Moser R, Silva Santos M de F, Luthi-Carter R. MicroRNA-22 (miR-22) overexpression is neuroprotective via general anti-apoptotic effects and may also target specific huntington's disease-related mechanisms. PLoS ONE 8(1), e54222 (2013).

48 Huang ZP, Chen J, Seok HY et al. MicroRNA-22 regulates cardiac hypertrophy and remodeling in response to stress. Circ. Res. 112(9), 1234-1243 (2013).

$49 \mathrm{Lu}$ W, You R, Yuan X et al. The microRNA miR-22 inhibits the histone deacetylase HDAC4 to promote TH17 celldependent emphysema. Nat. Immunol. 16(11), 1185-1194 (2015).

50 Han K, Gennarino VA, Lee Y et al. Human-specific regulation of MeCP2 levels in fetal brains by microRNA miR-483-5p. Genes Dev. 27(5), 485-490 (2013).

51 Li H, Xie H, Liu W et al. A novel microRNA targeting HDAC 5 regulates osteoblast differentiation in mice and contributes to primary osteoporosis in humans. J. Clin. Invest. 119(12), 3666-3677 (2009).

52 Fischer S, Paul AJ, Wagner A et al. miR-2861 as novel HDAC5 inhibitor in $\mathrm{CHO}$ cells enhances productivity while maintaining product quality. Biotechnol Bioeng. 112(10), 2142-2153 (2015).

53 Li CJ, Cheng P, Liang MK et al. MicroRNA-188 regulates age-related switch between osteoblast and adipocyte differentiation. J. Clin. Invest. 125(4), 1509-1522 (2015).

54 Nasser MW, Datta J, Nuovo G et al. Down-regulation of micro-RNA-1 (miR-1) in lung cancer: suppression of tumorigenic property of lung cancer cells and their sensitization to doxorubicin-induced apoptosis by miR-1. J. Biol. Chem. 283, 33394-33405 (2008).

55 Datta J, Kutay H, Nasser MW et al. Methylation mediated silencing of microRNA-1 gene and its role in hepatocellular carcinogenesis. Cancer Res. 68, 5049-5058 (2008).

56 Duan Z, Choy E, Nielsen GP et al. Differential expression of microRNA (miRNA) in chordoma reveals a role for miRNA-1 in Met expression. J. Orthop. Res. 6, 746-752 (2010).

57 Zhang J, Yang Y, Yang T et al. microRNA-22, downregulated in hepatocellular carcinoma and correlated with prognosis, suppresses cell proliferation and tumourigenicity. $B r . J$. Cancer 103, 1215-1220 (2010).

58 Song B, Wang Y, Xi Y et al. Mechanism of chemoresistance mediated by miR-140 in human osteosarcoma and colon cancer cells. Oncogene 28(46), 4065-4074 (2009).

59 Roccaro AM, Sacco A, Jia X et al. microRNA-dependent modulation of histone acetylation in Waldenstrom macroglobulinemia. Blood 116(9), 1506-1514 (2010).

60 Wilson AJ, Byun D-S, Nasser S et al. HDAC4 promotes growth of colon cancer cells via repression of p21. Mol. Biol. Cell 19, 4062-4075 (2008).

61 Mottet D, Pirotte S, Lamour V et al. HDAC4 represses p21(WAF1/Cip1) expression in human cancer cells through a Sp1-dependent, p53-independent mechanism. Oncogene 28, 243-256 (2009).

62 Yuan J hang, Yang F, Chen B feng et al. The histone deacetylase 4/SP1/microrna-200a regulatory network 
contributes to aberrant histone acetylation in hepatocellular carcinoma. Hepatology 54(6), 2025-2035 (2011).

Nishida N, Mimori K, Fabbri M et al. MicroRNA-125a-5p is an independent prognostic factor in gastric cancer and inhibits the proliferation of human gastric cancer cells in combination with trastuzumab. Clin. Cancer Res. 17(9), 2725-2733 (2011).

64 Sandhu SK, Volinia S, Costinean S et al. miR-155 targets histone deacetylase 4 (HDAC4) and impairs transcriptional activity of B-cell lymphoma 6 (BCL6) in the E $\mu$-miR-155 transgenic mouse model. Proc. Natl Acad. Sci. USA 109(49), 20047-20052 (2012).

65 Kai Y, Peng W, Ling W, Jiebing H, Zhuan B. Reciprocal effects between microRNA-140-5p and ADAM10 suppress migration and invasion of human tongue cancer cells. Biochem. Biophys. Res. Commun. 448, 308-314 (2014).

66 Wu MY, Fu J, Xiao X, Wu J, Wu RC. MiR-34a regulates therapy resistance by targeting HDAC1 and HDAC7 in breast cancer. Cancer Lett. 354(2), 311-319 (2014).

67 Paroni G, Mizzau M, Henderson C, Del Sal G, Schneider C, Brancolini C. Caspase-dependent regulation of histone deacetylase 4 nuclear-cytoplasmic shuttling promotes apoptosis. Mol. Biol. Cell 15, 2804-2818 (2004).

68 Scott FL, Fuchs GJ, Boyd SE et al. Caspase-8 cleaves histone deacetylase 7 and abolishes its transcription repressor function. J. Biol. Chem. 283, 19499-19510 (2008).

69 Liu F, Dowling M, Yang XJ, Kao GD. Caspase-mediated specific cleavage of human histone deacetylase 4. J. Biol. Chem. 279, 34537-34546 (2004).

70 Backs J, Worst BC, Lehmann LH et al. Selective repression of MEF2 activity by PKA-dependent proteolysis of HDAC4. J. Cell Biol. 195, 403-415 (2011).

71 Li X, Song S, Liu Y, Ko SH, Kao HY. Phosphorylation of the histone deacetylase 7 modulates its stability and association with 14-3-3 proteins. J. Biol. Chem. 279(33), 34201-34208 (2004).

72 Bradley EW, Carpio LR, Olson EN, Westendorf JJ. Histone deacetylase 7 (Hdac7) suppresses chondrocyte proliferation and $\beta$-catenin activity during endochondral ossification. J. Biol. Chem. 290, 118-126 (2015).

73 Arnold MA, Kim Y, Czubryt MP et al. MEF2C transcription factor controls chondrocyte hypertrophy and bone development. Dev. Cell. 12, 377-389 (2007).

74 Potthoff MJ, Wu H, Arnold MA et al. Histone deacetylase degradation and MEF2 activation promote the formation of slow-twitch myofibers. J. Clin. Invest. 117, 2459-2467 (2007).

75 Cernotta N, Clocchiatti A, Florean C, Brancolini C. Ubiquitin-dependent degradation of HDAC4, a new regulator of random cell motility. Mol. Biol. Cell. 22, 278-289 (2011).

76 Shimizu E, Nakatani T, He Z, Partridge NC. Parathyroid hormone regulates histone deacetylase (HDAC) 4 through protein kinase A-mediated phosphorylation and dephosphorylation in osteoblastic cells. J. Biol. Chem. 289, 21340-50 (2014).

77 Obri A, Makinistoglu MP, Zhang H, Karsenty G. HDAC4 integrates PTH and sympathetic signaling in osteoblasts. J. Cell Biol. 205(6), 771-780 (2014).
78 Guo L, Han A, Bates DL, Cao J, Chen L. Crystal structure of a conserved $\mathrm{N}$-terminal domain of histone deacetylase 4 reveals functional insights into glutamine-rich domains. Proc. Natl Acad. Sci. USA 104, 4297-4302 (2007).

79 Grégoire S, Tremblay AM, Xiao L et al. Control of MEF2 transcriptional activity by coordinated phosphorylation and sumoylation. J. Biol. Chem. 281, 4423-4433 (2006).

80 Gao C, Ho C-C, Reineke E et al. Histone deacetylase 7 promotes PML sumoylation and is essential for PML nuclear body formation. Mol. Cell. Biol. 28, 5658-5667 (2008).

81 Lee JH, Park SM, Kim OS et al. Differential SUMOylation of LXRalpha and LXRbeta mediates transrepression of STAT1 inflammatory signaling in IFN-gamma-stimulated brain astrocytes. Mol. Cell 35, 806-817 (2009).

82 Grozinger CM, Schreiber SL. Regulation of histone deacetylase 4 and 5 and transcriptional activity by 14-3-3-dependent cellular localization. Proc. Natl Acad. Sci. USA 97, 7835-7840 (2000).

83 Nishino TG, Miyazaki M, Hoshino H, Miwa Y, Horinouchi S, Yoshida M. 14-3-3 regulates the nuclear import of class IIa histone deacetylases. Biochem. Biophys. Res. Commun. 377, 852-856 (2008).

84 Paroni G, Cernotta N, Dello Russo C et al. PP2A regulates HDAC4 nuclear import. Mol. Biol. Cell. 19, 655-667 (2008).

85 Fischle W, Dequiedt F, Hendzel MJ et al. Enzymatic activity associated with class II HDACs is dependent on a multiprotein complex containing HDAC3 and SMRT/NCoR. Mol. Cell. 9, 45-57 (2002).

-• Shows the enzymatic ineptitude of vertebrates' class IIa histone deacetylases (HDACs). Class IIa HDACs acquired an HDAC/KDAC activity only if in complex with class I HDACs and it is therefore almost exclusively matter of the nucleus.

86 McKinsey TA, Zhang CL, Lu J, Olson EN. Signaldependent nuclear export of a histone deacetylase regulates muscle differentiation. Nature 408, 106-111 (2000).

-. In this paper for the first time it was demonstrated that the nuclear export of class IIa HDACs may influence physiological processes, such as muscle differentiation.

87 Backs J, Olson EN. Control of cardiac growth by histone acetylation/deacetylation. Circ. Res. 98, 15-24 (2006).

88 Martin M, Potente M, Janssens V et al. Protein phosphatase 2A controls the activity of histone deacetylase 7 during T cell apoptosis and angiogenesis. Proc. Natl Acad. Sci. USA 105(12), 4727-4732 (2008).

89 Kozhemyakina E, Cohen T, Yao T-P, Lassar AB. Parathyroid hormone-related peptide represses chondrocyte hypertrophy through a protein phosphatase $2 \mathrm{~A} /$ histone deacetylase 4/ MEF2 pathway. Mol. Cell. Biol. 29, 5751-5762 (2009).

90 Martin M, Kettmann R, Dequiedt F. Class IIa histone deacetylases: regulating the regulators. Oncogene 26, 5450-5467 (2007).

- Although this review dates back 2007, it provides an excellent overview of class IIa HDACs biology and principles of regulation. 
91 Wang Z, Qin G, Zhao TC. HDAC4: mechanism of regulation and biological functions. Epigenomics 6(1), 139-50 (2014).

$92 \mathrm{Li}$ C, Cai X, Sun $\mathrm{H}$ et al. The $\delta \mathrm{A}$ isoform of calmodulin kinase II mediates pathological cardiac hypertrophy by interfering with the HDAC4-MEF2 signaling pathway. Biochem. Biophys. Res. Commun. 409, 125-130 (2011).

93 Paroni G, Fontanini A, Cernotta N et al. Dephosphorylation and caspase processing generate distinct nuclear pools of histone deacetylase 4. Mol. Cell. Biol. 27, 6718-6732 (2007).

- In this article the function of different HDAC4 nuclear forms is investigated; up to now no other similar investigations have been made.

94 Walkinshaw DR, Weist R, Kim GW et al. The tumor suppressor kinase LKB1 activates the downstream kinases SIK2 and SIK3 to stimulate nuclear export of class IIa histone deacetylases. J. Biol. Chem. 288, 9345-9362 (2013).

-• In this paper authors overexpressed in HEK-293 cells various members of LKB1 kinase family, providing an excellent overview of the effects of the kinases of this family on class IIa HDACs export.

95 Backs J, Song K, Bezprozvannaya S, Chang S, Olson EN. CaM kinase II selectively signals to histone deacetylase 4 during cardiomyocyte hypertrophy. J. Clin. Invest. 116(7), 1853-1864 (2006).

96 Backs J, Backs T, Bezprozvannaya S, McKinsey TA, Olson EN. Histone deacetylase 5 acquires calcium/calmodulindependent kinase II responsiveness by oligomerization with histone deacetylase 4. Mol. Cell. Biol. 28, 3437-3445 (2008).

- Explains that certain class IIa HDACs that are not directly bound by a certain kinase, could become substrates of this kinase by complexing with another member of the family.

97 Bolger TA, Yao T-P. Intracellular trafficking of histone deacetylase 4 regulates neuronal cell death. J. Neurosci. 25, 9544-9553 (2005).

98 Shalizi A, Gaudillière B, Yuan Z et al. A calciumregulated MEF2 sumoylation switch controls postsynaptic differentiation. Science 311, 1012-1017 (2006).

99 Métrich M, Berthouze M, Morel E, Crozatier B, Gomez AM, Lezoualc'h F. Role of the cAMP-binding protein Epac in cardiovascular physiology and pathophysiology. Pflugers Arch. Eur. J. Physiol. 459, 535-546 (2010).

100 Métrich M, Laurent AC, Breckler M et al. Epac activation induces histone deacetylase nuclear export via a Ras-dependent signalling pathway. Cell. Signal. 22, 1459-1468 (2010).

101 Dequiedt F, Van Lint J, Lecomte E et al. Phosphorylation of histone deacetylase 7 by protein kinase $\mathrm{D}$ mediates $\mathrm{T}$ cell receptor-induced Nur77 expression and apoptosis. J. Exp. Med. 201, 793-804 (2005).

102 Matthews SA, Liu P, Spitaler M et al. Essential role for protein kinase D family kinases in the regulation of class II histone deacetylases in B lymphocytes. Mol Cell Biol. 26, 1569-1577 (2006).

103 Wilker PR, Kohyama M, Sandau MM et al. Transcription factor Mef2c is required for B cell proliferation and survival after antigen receptor stimulation. Nat. Immunol. 9, 603-612 (2008).
104 Parra M, Kasler H, McKinsey TA, Olson EN, Verdin E. Protein kinase D1 phosphorylates HDAC7 and induces its nuclear export after T-cell receptor activation. J. Biol. Chem. 280, 13762-13770 (2005).

105 Sin YY, Baillie GS. Protein kinase D in the hypertrophy pathway. Biochem. Soc. Trans. 40(1), 287-9 (2012).

$106 \mathrm{Xu} \mathrm{X}, \mathrm{Ha} \mathrm{CH}$, Wong $\mathrm{C}$ et al. Angiotensin II stimulates protein kinase D-dependent histone deacetylase 5 phosphorylation and nuclear export leading to vascular smooth muscle cell hypertrophy. Arterioscler. Thromb. Vasc. Biol. 27, 2355-2362 (2007).

107 Sinnett-Smith J, Ni Y, Wang J, Ming M, Young SH, Rozengurt E. Protein kinase D1 mediates class IIa histone deacetylase phosphorylation and nuclear extrusion in intestinal epithelial cells: role in mitogenic signaling. $\mathrm{Am}$. J. Physiol. Cell Physiol. 306(10), C961-C971 (2014).

108 Kim M-S, Fielitz J, McAnally J et al. Protein kinase D1 stimulates MEF2 activity in skeletal muscle and enhances muscle performance. Mol. Cell. Biol. 28, 3600-3609 (2008).

109 Hardie DG. AMP-activated protein kinase-an energy sensor that regulates all aspects of cell function. Genes Dev. 25(18), 1895-1908 (2011).

110 Dequiedt F, Martin M, Von Blume J et al. New role for hPar-1 kinases EMK and C-TAK1 in regulating localization and activity of class IIa histone deacetylases. Mol. Cell. Biol. 26, 7086-7102 (2006).

111 Bayraktar J, Zygmunt D, Carthew RW. Par-1 kinase establishes cell polarity and functions in Notch signaling in the Drosophila embryo. J. Cell Sci. 119, 711-721 (2006).

112 Guo S, Kemphues KJ. par-1, a gene required for establishing polarity in C. elegans embryos, encodes a putative Ser/Thr kinase that is asymmetrically distributed. Cell $81,611-620$ (1995).

113 Chen S, Yin C, Lao T et al. AMPK-HDAC5 pathway facilitates nuclear accumulation of HIF-1 $\alpha$ and functional activation of HIF-1 by deacetylating Hsp70 in the cytosol. Cell Cycle 14(15), 2520-36 (2015).

114 Zhao J-X, Yue W-F, Zhu M-J, Du M. AMP-activated protein kinase regulates beta-catenin transcription via histone deacetylase 5. J. Biol. Chem. 286(18), 16426-16434 (2011).

115 McGee SL, Fairlie E, Garnham AP, Hargreaves M. Exercise-induced histone modifications in human skeletal muscle. J. Physiol. 587(Pt 24), 5951-5958 (2009).

116 McGee SL, van Denderen BJW, Howlett KF et al. AMPactivated protein kinase regulates GLUT4 transcription by phosphorylating histone deacetylase 5 . Diabetes. 57 , 860-867 (2008).

117 McGee SL, Swinton C, Morrison S et al. Compensatory regulation of HDAC5 in muscle maintains metabolic adaptive responses and metabolism in response to energetic stress. FASEB J. 28(8), 3384-3395 (2014).

118 Katoh Y, Takemori H, Horike N et al. Salt-inducible kinase (SIK) isoforms: their involvement in steroidogenesis and adipogenesis. Mol. Cell. Endocrinol. 217(1-2), 109-112 (2004). 
119 Takemori H, Katoh Hashimoto Y, Nakae J, Olson EN, Okamoto M. Inactivation of HDAC5 by SIK1 in AICARtreated C2C12 myoblasts. Endocr. J. 56(1), 121-130 (2009).

120 Dietrich JB, Takemori H, Grosch-Dirrig S, Bertorello A, Zwiller J. Cocaine induces the expression of MEF2C transcription factor in rat striatum through activation of SIK1 and phosphorylation of the histone deacetylase HDAC5. Synapse 66(1), 61-70 (2012).

121 Finsterwald C, Carrard A, Martin JL. Role of salt-inducible kinase 1 in the activation of MEF2-dependent transcription by BDNF. PLoS ONE 8(1), e 54545 (2013).

122 Calnan DR, Brunet A. The FoxO code. Oncogene 27, 2276-2288 (2008).

123 Wang B, Moya N, Niessen S et al. A hormone-dependent module regulating energy balance. Cell 145, 596-606 (2011).

124 Henriksson E, Säll J, Gormand A et al. SIK2 regulates CRTCs, HDAC4 and glucose uptake in adipocytes. J. Cell Sci. 128, 472-486 (2015).

125 Luan B, Goodarzi MO, Phillips NG et al. Leptin-mediated increases in catecholamine signaling reduce adipose tissue inflammation via activation of macrophage HDAC4. Cell Metab. 19(6), 1058-1065 (2014).

126 Abu-Farha M, Tiss A, Abubaker J et al. Proteomics analysis of human obesity reveals the epigenetic factor HDAC4 as a potential target for obesity. PLoS ONE 8(9), e75342 (2013).

127 Choi S, Lim DS, Chung J. Feeding and fasting signals converge on the LKB1-SIK3 pathway to regulate lipid metabolism in drosophila. PLoS Genet. 11(5), e1005263 (2015).

128 Berdeaux R, Goebel N, Banaszynski L et al. SIK1 is a class II HDAC kinase that promotes survival of skeletal myocytes. Nat. Med. 13, 597-603 (2007).

129 Stewart R, Akhmedov D, Robb C, Leiter C, Berdeaux R. Regulation of SIK1 abundance and stability is critical for myogenesis. Proc. Natl Acad. Sci. USA 110(1), 117-122 (2013).

130 van der Linden AM, Nolan KM, Sengupta P. KIN-29 SIK regulates chemoreceptor gene expression via an MEF2 transcription factor and a class II HDAC. EMBO J. 26, $358-370$ (2007).

$131 \mathrm{Li} \mathrm{J}$, Jiang D. The role of epigenomics in the neurodegeneration of ataxia-telangiectasia. Epigenomics 7(2), 137-141 (2015).

132 Li J, Chen J, Ricupero CL et al. Nuclear accumulation of HDAC4 in ATM deficiency promotes neurodegeneration in ataxia telangiectasia. Nat. Med. 18, 783-790 (2012).

133 Parra M, Mahmoudi T, Verdin E. Myosin phosphatase dephosphorylates HDAC7, controls its nucleocytoplasmic shuttling, and inhibits apoptosis in thymocytes. Genes Dev. 21, 638-643 (2007).

134 Chang S, McKinsey TA, Zhang CL, Richardson JA, Hill JAOE. Histone deacetylases 5 and 9 govern responsiveness of the heart to a subset of stress signals and play redundant roles in heart development. Mol. Cell. Biol. 24, 8467-8476 (2004).
135 Perry RLS, Yang C, Soora N et al. Direct interaction between myocyte enhancer factor 2 (MEF2) and protein phosphatase 1alpha represses MEF2-dependent gene expression. Mol. Cell. Biol. 29, 3355-3366 (2009).

136 Greco TM, Yu F, Guise AJ, Cristea IM. Nuclear import of histone deacetylase 5 by requisite nuclear localization signal phosphorylation. Mol. Cell. Proteomics. 10(2), M110.004317 (2011).

137 Deng X, Ewton DZ, Mercer SE, Friedman E. Mirk/dyrk1B decreases the nuclear accumulation of class II histone deacetylases during skeletal muscle differentiation. J. Biol. Chem. 280, 4894-4905 (2005).

$138 \mathrm{Ha} \mathrm{CH}$, Kim JY, Zhao J et al. PKA phosphorylates histone deacetylase 5 and prevents its nuclear export, leading to the inhibition of gene transcription and cardiomyocyte hypertrophy. Proc. Natl Acad. Sci. USA 107, 15467-15472 (2010).

139 Taniguchi M, Carreira MB, Smith LN, Zirlin BC, Neve RL, Cowan CW. Histone deacetylase 5 limits cocaine reward through camp-induced nuclear import. Neuron 73, 108-120 (2012).

140 Ago T, Liu T, Zhai P et al. A redox-dependent pathway for regulating class II HDACs and cardiac hypertrophy. Cell 133, 978-993 (2008).

- In this paper HDAC4 was proposed for the first time as a redox-sensor. Despite many authors confirmed these evidences, up to now it is not clear if this redox-sensitivity is a general or a context-dependent property of HDAC4.

141 Di Giorgio E, Gagliostro E, Brancolini C. Selective class IIa HDAC inhibitors: myth or reality. Cell. Mol. Life Sci. 72, 73-86 (2015).

142 Hudson GM, Watson PJ, Fairall L, Jamieson AG, Schwabe JW. Insights into the recruitment of class IIa histone deacetylases (HDACs) to the SMRT/NCoR transcriptional repression complex. J. Biol. Chem. 290, 18237-18244 (2015).

- In this paper for the first time a proteomic approach has been used to investigate the nature of the class IIa HDACsSMRT/NCoR repressive complex.

143 Oka SI, Ago T, Kitazono T, Zablocki D, Sadoshima J. The role of redox modulation of class II histone deacetylases in mediating pathological cardiac hypertrophy. J. Mol. Med. 87, 785-791 (2009).

144 Haworth RS, Stathopoulou K, Candasamy AJ, Avkiran M. Neurohormonal regulation of cardiac histone deacetylase 5 nuclear localization by phosphorylation-dependent and phosphorylation-independent mechanisms. Circ. Res. 110(12), 1585-1595 (2012).

145 Du M, Perry RLS, Nowacki NB et al. Protein kinase A represses skeletal myogenesis by targeting myocyte enhancer factor 2D. Mol. Cell. Biol. 28, 2952-2970 (2008).

146 Guise AJ, Greco TM, Zhang IY, Yu F, Cristea IM. Aurora B-dependent regulation of class IIa histone deacetylases by mitotic nuclear localization signal phosphorylation. Mol. Cell. Proteomics 11, 1220-1229 (2012). 